\title{
Effects of Nigella sativa on Performance, Blood Profiles, and Antibody Titer against Newcastle Disease in Broilers
}

\author{
Alireza Talebi $\left(\mathbb{D},{ }^{1}\right.$ Masoud Maham $\left(\mathbb{D},{ }^{2}\right.$ Siamak Asri-Rezaei ${ }^{(D)},{ }^{2}$ Pouya Pournaghi $\left(\mathbb{D},{ }^{3,4}\right.$ \\ Mohammad-Sadegh Khorrami, ${ }^{3}$ and Amir Derakhshan ${ }^{3}$ \\ ${ }^{1}$ Department of Poultry Health \& Diseases, Faculty of Veterinary Medicine, Urmia University, \\ P.O. Box 57153-1177tbox57153-1177, Urmia, Iran \\ ${ }^{2}$ Department of Large Animal Medicine, Faculty of Veterinary Medicine, Urmia University, P.O. Box 57153-1177, Urmia, Iran \\ ${ }^{3}$ Graduated of Veterinary College, Urmia University, Urmia, Iran \\ ${ }^{4}$ Department of Biology, Payame Noor University, Tehran, Iran
}

Correspondence should be addressed to Alireza Talebi; a.talebi@urmia.ac.ir

Received 10 April 2021; Accepted 31 May 2021; Published 14 June 2021

Academic Editor: Valeria Sülsen

Copyright @ 2021 Alireza Talebi et al. This is an open access article distributed under the Creative Commons Attribution License, which permits unrestricted use, distribution, and reproduction in any medium, provided the original work is properly cited.

\begin{abstract}
Recent anxiety about resistance to chemical drugs has elevated the position of phytogenic feed additives including Nigella sativa in preventive strategy in the poultry industry. During this study, a completely randomized experiment was designed to investigate the efficacy of different levels ( 0 to $16 \%$ ) of $N$. sativa seeds supplemented in the diet of broilers on performance, immune responses, and hematological and biochemical parameters. The results indicated the following: (a) Supplementation of $1 \%$ N. sativa seeds in diet had the highest positive effects and $16 \%$ N. sativa had the highest significant $(p=0.03)$ adverse effects on weight gain, while up to $2 \%$ N. sativa seeds in the diet reduced feed conversion ratio (FCR) whereas $4 \%$ and over that increased the FCR. (b) Chickens fed with a diet containing $1 \%$ N. sativa seeds had the highest antibody titers, but those fed with $16 \%$ N. sativa seeds had the lowest antibody titers at end of the experiment. (c) Dietary inclusion of $N$. sativa seeds increased hemogram parameters and the group fed with $16 \%$ N. sativa seeds had the highest values on day 21 until the end of the experiment. (d) Supplementation of $N$. sativa seeds decreased in WBC and lymphocytes but increased heterophils, H/L, monocytes, eosinophils, and basophils percentages. Supplementation of up to $2 \%$ of $N$. sativa seeds in broiler's diets elaborated serum level of those parameters, while supplementation of $\geq 4 \%$ N. sativa seeds decreased their serum levels. In conclusion, supplementation of N. sativa seed (1-2\%) in broiler diets, as a multipurpose natural growth promoter, improves performance, elevates humoral immune responses, affects serum biochemical profiles of broiler chickens, and induces changes in their hemogram and leukogram, while there are no side, residual, and hazardous effects.
\end{abstract}

\section{Introduction}

Phytogenic feed additives "phytobiotic" are assuming a position of prime importance in the poultry industry [1-3] and could be used in feed or water [4]. Among the promising phytogenic immune-stimulants, Nigella sativa (N. sativa) is a miracle medicinal herb having a long history with a rich religious background $[5,6]$. N. sativa seeds are used for the treatment of some disorders and illnesses including fever, common cold, headache, asthma, various Gram-positive and Gram-negative microbial infections [7], aflatoxicosis [8], antiethanol hepatotoxicity [9], and alleviation of adverse effects of heat stress [10], as well as to expel worms from the intestines. The possible biochemical active components of $N$. sativa and their pharmacological effects have been investigated $[11,12]$, and their pharmacological actions as immune stimulation, antioxidant, anti-inflammatory, antimicrobial, and antiparasitic activities [5, 13-17] have also been studied. Recently, the potential effects of $N$. sativa seeds' bioactive compounds (such as thymoquinone, $\alpha$-hederin, and nigellidine) in control of the COVID-19 pandemic have also been documented [18-20]. In the production of healthy food, 
supplementation of $N$. sativa seeds to poultry diets could be recommended as a natural growth promoter instead of antibiotics. Previous reports indicate that diets containing different levels of $N$. sativa seeds improve the performance and carcass characteristics in broilers $[5,21]$. The inclusion of $N$. sativa seeds in layers' diet also increases egg production as well as eggs' quality [22] and serum triglycerides in layers [23]. However, N. sativa also affects blood profile and biochemical parameters $[24,25]$ and prevents atherogenesis by decreasing LDL-cholesterol and increasing HDL cholesterol [26]. N. sativa can control infectious diseases and regulate adaptive immunity via enhancement of macrophages [27]. Effects of N. sativa on cell-mediated immunity [28] and humoral immunity [29] have also been investigated. Although it has been documented that $N$. sativa has immunomodulatory effects [5], the type of immune responses against various pathogens provoked by $N$. sativa may need further investigations. However, interest in herbal medicines and their multiple beneficial utilization in poultry health and performance has been recently reviewed [5, 6, 30-32]. Finding the best beneficial and safe phytogenic feed additives as the most suitable substitute to antibiotic promoters is much more today's research topic [1, 2, 4, 33-35].

The objective of this research was a comprehensive study on the efficacy of $N$. sativa as a multipurpose feed additive on performance, blood profiles, and humoral immune responses in broiler chickens. To the best of our knowledge, the closest reports to our study in the literature are works of Al-Beitawi et al. [29], Durrani et al. [36], Shewita and Taha [37], Ghasemi et al. [38], Hossain et al. [39], Shirzadegan et al. [40], Singh and Kumar [41], and Aydogan et al. [42], and the present study has a lot of differences with them including the use of various percentages of $N$. sativa seeds in diets and weekly measurement of performance, immune responses, serum biochemical parameters, hemogram, and leukogram indices.

\section{Materials and Methods}

2.1. Ethics of Experimentation. All experimental procedures were carried out according to the standard animal experimentation protocols of the Veterinary Ethics Committee of Faculty of Veterinary Medicine, Urmia University (Ref. IRUU-AEC-3/902/DA).

2.2. Experimental Design and Chicken Management. In a completely randomized experiment, one hundred sixty unsexed one-day-old chicks (Ross-308) were divided into 8 treatment groups (20 chicks/group) with 4 replicates. The treatment groups were $\mathrm{T} 0=$ control 1 (without vaccination and without supplementation of $N$. sativa in diet), T1 $=$ control 2 $(\mathrm{T} 0+$ Vaccination), $\mathrm{T} 2=\mathrm{T} 1+N$. sativa $0.5 \%, \quad \mathrm{~T} 3=\mathrm{T} 1+N$. sativa $1 \%, \mathrm{~T} 4=\mathrm{T} 1+N$. sativa $2 \%, \mathrm{~T} 5=\mathrm{T} 1+N$. sativa $4 \%$, $\mathrm{T} 6=\mathrm{T} 1+N$. sativa $8 \%$, and $\mathrm{T} 7=\mathrm{T} 1+N$. sativa $16 \%$. After leg labeling, the chicks were housed in separated boxes, and environmental conditions (ambient temperature, humidity, lighting, ventilation, nutrition, etc.) are provided according to the technical instructions of Ross-308 for the broiler management guide. The basal diets (Table 1) were prepared according to the Ross-308 broiler nutritional specification guide. Energy and protein of $N$. sativa seed treated (T2-T7) groups' diets were roughly balanced by reducing $0.1 \mathrm{Kg}$ oil + 0.47 Kg corn + 0.43 Kg soybean meal for supplementation of each $1 \mathrm{Kg}$ of $\mathrm{N}$. sativa seed.

2.3. Vaccines and Vaccination Routes. According to breakthrough level 1:8 $\left(\log 2^{-3}\right)$ of maternal antibody [43], live ND clone 30 vaccine (eye-drop route) together with ND oil (subcutaneous route) is used for primary vaccination on day 9 of age, and the chickens were revaccinated with live ND clone-30 vaccine by eye-drop method [44] on the 21 st day.

2.4. Sampling Procedures. Blood samples for hematological tests (using EDTA anticoagulant-treated syringes) and blood samples for evaluation of immunity and biochemical tests were collected on day one and then at weekly intervals until the end of the experimental period (42 days old), as previously described [45]. Blood samples were labeled according to the number and date of sampling and kept at room temperature until clotted, and then, the sera were separated for serological tests.

2.5. Evaluation of Performance. The performance of the chickens for each week was determined.

2.6. Evaluation of Antibody Titer. On day one and day seven of age, serum samples were used to evaluate maternally derived antibodies of the chicks to determine the best time of first vaccination. On day 14 and weekly interval, serum samples were used to assess protective immune response derived from vaccination against ND by application of hemagglutination inhibition (HI) test as it has been reported that the HI test is an excellent indicator of the immune status and disease resistance of a flock, especially to assess protective responses following vaccination because, unlike ELISA, HI test correlates well with the more laborious virus neutralization (VN) assays [44].

2.7. Hematological Tests. Hematological parameters such as red blood cell (RBC) counts, PCV, hemoglobin $(\mathrm{Hb})$ concentration, white blood cells (WBC) counts, and percentages of heterophils, lymphocytes, monocytes, eosinophils, basophils, and heterophils to lymphocytes $(\mathbf{H} / \mathbf{L})$ ratio were determined by routine methods as previously described [46].

2.8. Biochemical Tests. Serum biochemical parameters values were determined by using an autoanalyzer (Technicon RA 1000 TM, Hartwell, LA, USA) and specific Pars-Azmun kits (Pars-Azmun Co., Tehran, Iran) as follows: albumin (ALB) concentration $(\mathrm{mg} / \mathrm{dL}$ with the bromcresol green method at $546 \mathrm{~nm}$ WL), alkaline phosphatase (ALP) concentration (U/ $\mathrm{L}$ with DGKC method at $405 \mathrm{~nm} \mathrm{WL}$ ), alanine transaminase (ALT), or serum glutamate-pyruvate transaminase (SGPT) concentration (U/L with Opt. standard method, IFCC at 
TABLE 1: Ingredients and nutrients of the basal diet.

\begin{tabular}{|c|c|c|c|}
\hline Ingredients (E kcal, CP \%) & Starter (1-10 days) & Grower (11-10 day 25 days) & Finisher $>26$ days) \\
\hline Corn $(3350,8.9)$ & $51 \%$ & $42 \%$ & $36 \%$ \\
\hline Soya meal $(2300,44)$ & $42 \%$ & $35.5 \%$ & $32.4 \%$ \\
\hline Wheat $(2900,12)$ & - & $10 \%$ & $14 \%$ \\
\hline Barley $(2600,11)$ & - & $4 \%$ & $8 \%$ \\
\hline Oil $(9000,0)$ & $3.3 \%$ & $5.4 \%$ & $7 \%$ \\
\hline Oyster shell & $1.6 \%$ & $1.4 \%$ & $1.2 \%$ \\
\hline Monocalcium phosphate & $1.3 \%$ & $1.2 \%$ & $1.1 \%$ \\
\hline Vitamin premix & $0.25 \%$ & $0.25 \%$ & $0.25 \%$ \\
\hline Mineral premix & $0.25 \%$ & $0.25 \%$ & $0.25 \%$ \\
\hline $\mathrm{Cl} \mathrm{Na}$ & $0.15 \%$ & $0.15 \%$ & $0.15 \%$ \\
\hline DKAB complex & $0.1 \%$ & $0.05 \%$ & $0.05 \%$ \\
\hline L-Lysin & $0.1 \%$ & $0.08 \%$ & $0.08 \%$ \\
\hline DL-Methionin & $0.1 \%$ & $0.1 \%$ & $0.1 \%$ \\
\hline N. sativa seed $(3450,23)$ & 0 & 0 & 0 \\
\hline Total & 100.15 & 100.33 & 100.58 \\
\hline \multicolumn{4}{|c|}{$\begin{array}{l}\text { Energy and protein of } 1 \mathrm{~kg} N \text {. sativa seeds were simply considered equal to nutrients of } 0.47 \mathrm{~kg} \text { corn }+0.43 \mathrm{~kg} \text { soya meal }+0.1 \mathrm{~kg} \text { oil; } \\
\text { therefore, for each } 1 \mathrm{~kg} \text { of } N \text {. sativa seeds supplementation, } 0.47 \mathrm{~kg} \text { corn }+0.43 \mathrm{~kg} \text { soya meal }+0.1 \mathrm{~kg} \text { oil were reduced. } \\
\text { Calculate major nutrients }\end{array}$} \\
\hline Energy (E) kcal/KgF & 3001.5 & 3103.5 & 3202.8 \\
\hline Protein (CP) \% & 23.02 & 21.0 & 20.02 \\
\hline $\mathrm{E} / \mathrm{CP}$ & 130.35 & 147.66 & 160.14 \\
\hline Calcium \% & 0.973 & 0.865 & 0.802 \\
\hline Phosphorus \% & 0.448 & 0.44 & 0.40 \\
\hline
\end{tabular}

$340 \mathrm{~nm}$ WL), aspartate transaminase (AST) or serum glutamic oxaloacetic transaminase (SGOT) concentration (U/L with Opt. standard method, IFCC at $340 \mathrm{~nm} \mathrm{WL}$ ), calcium concentration $(\mathrm{mg} / \mathrm{dL}$ with cresolphthalein complex method at 550-590 nm WL), cholesterol concentration (mg/dl with chod-pap method at $546 \mathrm{~nm} \mathrm{WL}$ ), glucose concentration (mg/dL with god-pap method at 500-546 nm WL), phosphorus concentration $(\mathrm{mg} / \mathrm{dL}$ with $\mathrm{UV}$ method at $340 \mathrm{~nm}$ $\mathrm{WL})$, total protein concentration $(\mathrm{mg} / \mathrm{dL}$ with Biuret method at 540-546 $\mathrm{nm} \mathrm{WL}$ ), and triglyceride concentration (mg/dL with gpo-pap enzymatic method at $546 \mathrm{~nm} \mathrm{WL})$.

2.9. Statistical Analysis. The results were analyzed by using the SPSS software (Version 21; SPSS Inc., Chicago, USA). The means for the treatments showing significant differences in the ANOVA were compared using the Post Hoc Tukey and Wilcoxon (nonparametric tests, 2 related samples) tests used for analyzing data of each parameter within the group and between groups. The relationship between age and parameter contents was ascertained through the Spearman correlation coefficient test. Differences were considered significant at $p<0.05$.

\section{Results}

3.1. Performance. N. sativa had some effects on the performance of the chickens (Table 2), and as shown in Figure 1, the chickens of group T3 fed with a diet containing 1\% $N$. sativa seeds had the highest weight at the end of the experiment but differences with those of the groups (T0-T5) were not significant $(p>0.05)$, but those with those of the groups (T6 and T7) were significant $(p<0.05)$. Dietary inclusion of $16 \% N$. sativa seeds had a significant $(p<0.03)$ depressive effect on weight gain of chickens (T7) during the experimental period. As shown in Table 2, the feed efficiency ratio was affected by the level of $N$. sativa seeds, and supplementation (T3 and T4) of the diet with $1 \%-2 \%$ N. sativa seeds had decreased FCR but $8 \%-16 \%$ increased FCR.

3.2. Immune Response. As shown in Figure 2, maternal antibody titer (MDA) of the chickens against ND decreased gradually and reached the points that are considered as negative in the control group T0 (environmental contamination control) on day 21 of age, and the comparison of average antibody titers of the groups during 2 weeks of age could be explained where maternal antibody of the chick's yolk may contribute to MDA of chicks during 3 days of age, and reduction of the MDA starts on day 4 of age based on half-life time (4-5 days) in case of ND [42]. On the contrary, antibody titers of ND of the vaccinated chickens (group T1-T7) were increased after vaccination at the 14th day of the experiment. Different levels of $N$. sativa seeds in diet affected antibody titers against ND in the vaccinated groups and the chickens (group T3) fed with $1 \% N$. sativa supplementation had the highest level of antibody titers, while chickens of group T7 (16\% N. sativa seed in diet) had the lowest level of antibody titers on days 28, 35, and 42 of age (Figure 2). Weekly comparison of antibody titers of the groups revealed the following: (1) Differences among the groups were not significant $(p<0.05)$ on days 1,7 , and 14 of 
TABLE 2: Effects of $N$. sativa on weekly performance (mean \pm SEM) of broilers.

\begin{tabular}{|c|c|c|c|c|c|c|}
\hline Age (day) & Group & Weight $(\mathrm{g})$ & Weight gain $(\mathrm{g})$ & Feed (g/week) & FCR (weekly) & FCR (total) \\
\hline \multirow[t]{4}{*}{ Day 1} & T0-T7 & $39.64 \pm 0.08 \mathrm{NS}$ & - & - & - & - \\
\hline & T0 & $167.5 \pm 5.6 a$ & $128.0 \pm 5.4 \mathrm{a}$ & $205 \pm 5.5$ & $1.60 \pm 0.07 a$ & $1.22 \pm 0.04 \mathrm{a}$ \\
\hline & $\mathrm{T} 1$ & $157.8 \pm 6.5 a$ & $118.6 \pm 4.6 \mathrm{~b}$ & $195 \pm 4.6$ & $1.65 \pm 0.05 b$ & $1.23 \pm 0.05 \mathrm{a}$ \\
\hline & $\mathrm{T} 2$ & $157.1 \pm 5.8 \mathrm{a}$ & $117.4 \pm 4.7 \mathrm{bc}$ & $190 \pm 4.0$ & $1.62 \pm 0.03 \mathrm{ab}$ & $1.21 \pm 0.03 \mathrm{a}$ \\
\hline \multirow{8}{*}{ Day 7} & $\mathrm{~T} 3$ & $160.4 \pm 6.2 \mathrm{a}$ & $120.8 \pm 5.2 b$ & $193 \pm 3.5$ & $1.60 \pm 0.04 \mathrm{a}$ & $1.20 \pm 0.04 \mathrm{a}$ \\
\hline & $\mathrm{T} 4$ & $157.8 \pm 7.5 a$ & $118.0 \pm 4.6 \mathrm{bc}$ & $189 \pm 4.0$ & $1.60 \pm 0.05 a$ & $1.20 \pm 0.05 \mathrm{a}$ \\
\hline & T5 & $151.3 \pm 6.0 \mathrm{a}$ & $111.7 \pm 5.0 c$ & $181 \pm 3.7$ & $1.62 \pm 0.06 \mathrm{ab}$ & $1.20 \pm 0.06 \mathrm{a}$ \\
\hline & T6 & $146.0 \pm 3.5 \mathrm{ab}$ & $106.3 \pm 6.2 \mathrm{dc}$ & $175 \pm 4.0$ & $1.65 \pm 0.05 b$ & $1.20 \pm 0.04 \mathrm{a}$ \\
\hline & $\mathrm{T} 7$ & $137.0 \pm 3.4 b$ & $97.3 \pm 4.0 \mathrm{e}$ & $175 \pm 4.5$ & $1.80 \pm 0.06 c$ & $1.27 \pm 0.05 \mathrm{a}$ \\
\hline & T0 & $417.8 \pm 19.2 \mathrm{a}$ & $250.3 \pm 11.0 \mathrm{a}$ & $451 \pm 15.0$ & $1.80 \pm 0.07 \mathrm{a}$ & $1.57 \pm 0.06 \mathrm{a}$ \\
\hline & $\mathrm{T} 1$ & $391.7 \pm 11.9 \mathrm{a}$ & $234.0 \pm 12.5 \mathrm{bd}$ & $440 \pm 16.5$ & $1.88 \pm 0.06 \mathrm{ab}$ & $1.62 \pm 0.05 \mathrm{ac}$ \\
\hline & $\mathrm{T} 2$ & $406.3 \pm 19.1 \mathrm{a}$ & $249.2 \pm 13.0 \mathrm{ca}$ & $461 \pm 20.0$ & $1.85 \pm 0.06 \mathrm{ab}$ & $1.60 \pm 0.06 \mathrm{ac}$ \\
\hline \multirow{8}{*}{ Day 14} & $\mathrm{~T} 3$ & $426.0 \pm 26.9 \mathrm{a}$ & $265.6 \pm 12.8 c$ & $483 \pm 19.0$ & $1.82 \pm 0.07 \mathrm{ab}$ & $1.58 \pm 0.07 \mathrm{ba}$ \\
\hline & $\mathrm{T} 4$ & $424.8 \pm 21.3 \mathrm{a}$ & $267.0 \pm 13.2 c$ & $480 \pm 17.5$ & $1.80 \pm 0.06 a$ & $1.58 \pm 0.06 \mathrm{ba}$ \\
\hline & $\mathrm{T} 5$ & $420.0 \pm 16.3 a$ & $268.7 \pm 14.0 \mathrm{c}$ & $483 \pm 18.0$ & $1.80 \pm 0.08 \mathrm{a}$ & $1.59 \pm 0.05 \mathrm{ac}$ \\
\hline & T6 & $388.4 \pm 16.4 \mathrm{a}$ & $242.4 \pm 12.3 \mathrm{da}$ & $460 \pm 19.0$ & $1.90 \pm 0.07 b c$ & $1.63 \pm 0.04 \mathrm{ac}$ \\
\hline & $\mathrm{T} 7$ & $316.0 \pm 16.1 b$ & $179.0 \pm 11.0 \mathrm{e}$ & $349 \pm 16.5$ & $1.95 \pm 0.08 c$ & $1.66 \pm 0.05 c$ \\
\hline & T0 & $819.0 \pm 50.0 \mathrm{a}$ & $401.2 \pm 21.0 \mathrm{a}$ & $674 \pm 32.0$ & $1.68 \pm 0.06 \mathrm{ab}$ & $1.62 \pm 0.04 \mathrm{a}$ \\
\hline & $\mathrm{T} 1$ & $774.7 \pm 27.0 \mathrm{ab}$ & $383.0 \pm 19.5 b$ & $648 \pm 29.0$ & $1.69 \pm 0.07 \mathrm{ab}$ & $1.65 \pm 0.06 \mathrm{ab}$ \\
\hline & $\mathrm{T} 2$ & $784.1 \pm 54.0 \mathrm{ab}$ & $377.8 \pm 17.5 b$ & $627 \pm 28.0$ & $1.66 \pm 0.05 \mathrm{a}$ & $1.63 \pm 0.05 a$ \\
\hline \multirow{8}{*}{ Day 21} & $\mathrm{~T} 3$ & $828.8 \pm 70.0 \mathrm{a}$ & $402.8 \pm 18.0 \mathrm{a}$ & $665 \pm 27.5$ & $1.65 \pm 0.06 \mathrm{a}$ & $1.61 \pm 0.04 a$ \\
\hline & $\mathrm{T} 4$ & $794.1 \pm 28.0 \mathrm{ab}$ & $369.5 \pm 16.9 c$ & $609 \pm 25.0$ & $1.65 \pm 0.04 \mathrm{a}$ & $1.61 \pm 0.06 \mathrm{a}$ \\
\hline & $\mathrm{T} 5$ & $783.5 \pm 26.0 \mathrm{ab}$ & $363.0 \pm 16.4 c$ & $607 \pm 24.5$ & $1.67 \pm 0.06 \mathrm{ab}$ & $1.62 \pm 0.04 \mathrm{a}$ \\
\hline & T6 & $703.5 \pm 24.0 \mathrm{bc}$ & $315.1 \pm 15.0 \mathrm{~d}$ & $541 \pm 26.8$ & $1.74 \pm 0.06 \mathrm{bc}$ & $1.68 \pm 0.05 \mathrm{ab}$ \\
\hline & $\mathrm{T} 7$ & $611.9 \pm 24.0 c$ & $295.9 \pm 13.8 \mathrm{e}$ & $523 \pm 24.5$ & $1.77 \pm 0.08 c$ & $1.71 \pm 0.07 b$ \\
\hline & T0 & $1315.0 \pm 40 \mathrm{a}$ & $496.0 \pm 24.0 \mathrm{a}$ & $808 \pm 26.0$ & $1.63 \pm 0.05 \mathrm{ab}$ & $1.62 \pm 0.06 \mathrm{ab}$ \\
\hline & $\mathrm{T} 1$ & $1266.4 \pm 36 a$ & $491.7 \pm 24.8 \mathrm{a}$ & $807 \pm 27.0$ & $1.64 \pm 0.05 \mathrm{ab}$ & $1.64 \pm 0.07 \mathrm{ab}$ \\
\hline & $\mathrm{T} 2$ & $1274.4 \pm 64 \mathrm{a}$ & $490.3 \pm 26.0 \mathrm{a}$ & $800 \pm 25.0$ & $1.63 \pm 0.06 \mathrm{ab}$ & $1.63 \pm 0.04 \mathrm{ab}$ \\
\hline \multirow{8}{*}{ Day 28} & $\mathrm{~T} 3$ & $1322.6 \pm 61 a$ & $493.8 \pm 25.5 a$ & $780 \pm 24.5$ & $1.58 \pm 0.05 b$ & $1.60 \pm 0.06 a$ \\
\hline & $\mathrm{T} 4$ & $1277.5 \pm 60 \mathrm{a}$ & $483.4 \pm 24.0 \mathrm{a}$ & $778 \pm 22.6$ & $1.60 \pm 0.07 \mathrm{ab}$ & $1.60 \pm 0.07 \mathrm{a}$ \\
\hline & T5 & $1250.4 \pm 37 b$ & $466.9 \pm 24.3 b$ & $779 \pm 24.0$ & $1.67 \pm 0.06 \mathrm{ab}$ & $1.64 \pm 0.05 \mathrm{ab}$ \\
\hline & T6 & $1076.4 \pm 57 \mathrm{bc}$ & $372.9 \pm 23.5 c$ & $624 \pm 26.0$ & $1.68 \pm 0.07 \mathrm{ca}$ & $1.68 \pm 0.06 b$ \\
\hline & $\mathrm{T} 7$ & $921.0 \pm 47 c$ & $309.1 \pm 22.5 \mathrm{~d}$ & $572 \pm 25.0$ & $1.85 \pm 0.08 \mathrm{~d}$ & $1.75 \pm 0.07 c$ \\
\hline & T0 & $1919.0 \pm 39 a$ & $604.0 \pm 35.0 \mathrm{a}$ & $1056 \pm 33.0$ & $1.70 \pm 0.07 \mathrm{a}$ & $1.64 \pm 0.05 a$ \\
\hline & $\mathrm{T} 1$ & $1840.6 \pm 36 a$ & $574.2 \pm 29.5 \mathrm{ab}$ & $993 \pm 29.0$ & $1.73 \pm 0.07 a$ & $1.67 \pm 0.05 \mathrm{ab}$ \\
\hline & $\mathrm{T} 2$ & $1871.0 \pm 44 \mathrm{a}$ & $596.6 \pm 26.0 \mathrm{a}$ & $1032 \pm 35.0$ & $1.73 \pm 0.06 a$ & $1.66 \pm 0.06 \mathrm{ab}$ \\
\hline \multirow{8}{*}{ Day 35} & $\mathrm{~T} 3$ & $1925.0 \pm 41 \mathrm{a}$ & $602.4 \pm 28.0 \mathrm{a}$ & $1036 \pm 32.0$ & $1.72 \pm 0.05 \mathrm{a}$ & $1.64 \pm 0.04 \mathrm{a}$ \\
\hline & $\mathrm{T} 4$ & $1880.0 \pm 61 \mathrm{a}$ & $602.5 \pm 32.0 \mathrm{a}$ & $1043 \pm 38.5$ & $1.73 \pm 0.07 a$ & $1.66 \pm 0.05 \mathrm{ab}$ \\
\hline & T5 & $1808.0 \pm 39 a$ & $557.6 \pm 28.5 b c$ & $1003 \pm 35.0$ & $1.80 \pm 0.08 b$ & $1.69 \pm 0.07 \mathrm{ab}$ \\
\hline & T6 & $1623.0 \pm 34 b$ & $548.6 \pm 27.0 \mathrm{bc}$ & $999 \pm 38.0$ & $1.82 \pm 0.07 b c$ & $1.72 \pm 0.07 b$ \\
\hline & $\mathrm{T} 7$ & $1454.6 \pm 63 c$ & $533.6 \pm 25.0 c$ & $997 \pm 28.0$ & $1.87 \pm 0.08 c$ & $1.80 \pm 0.08 c$ \\
\hline & T0 & $2476.2 \pm 33 a$ & $557.2 \pm 28.0 \mathrm{a}$ & $1131 \pm 35.0$ & $2.03 \pm 0.09 a$ & $1.73 \pm 0.07 a$ \\
\hline & $\mathrm{T} 1$ & $2412.1 \pm 37 a$ & $571.5 \pm 26.5 a$ & $1171 \pm 36.0$ & $2.05 \pm 0.08 \mathrm{a}$ & $1.76 \pm 0.08 \mathrm{a}$ \\
\hline & $\mathrm{T} 2$ & $2436.0 \pm 62 a$ & $565.0 \pm 24.0 \mathrm{a}$ & $1164 \pm 38.0$ & $2.06 \pm 0.09 \mathrm{a}$ & $1.75 \pm 0.08 \mathrm{a}$ \\
\hline \multirow{5}{*}{ Day 42} & $\mathrm{~T} 3$ & $2586.0 \pm 57 a$ & $661.0 \pm 29.5 b$ & $1348 \pm 41.0$ & $2.04 \pm 0.07 \mathrm{a}$ & $1.74 \pm 0.07 \mathrm{a}$ \\
\hline & $\mathrm{T} 4$ & $2447.6 \pm 64 a$ & $567.5 \pm 26.5 a$ & $1191 \pm 37.0$ & $2.10 \pm 0.08 \mathrm{ab}$ & $1.75 \pm 0.07 a$ \\
\hline & T5 & $2352.8 \pm 64 a$ & $544.8 \pm 26.0 \mathrm{a}$ & $1183 \pm 33.0$ & $2.17 \pm 0.09 b$ & $1.79 \pm 0.06 \mathrm{ab}$ \\
\hline & T6 & $2115.1 \pm 57 b$ & $492.1 \pm 24.5 c$ & $1082 \pm 36.0$ & $2.20 \pm 0.09 b$ & $1.84 \pm 0.05 b c$ \\
\hline & $\mathrm{T} 7$ & $1944.2 \pm 42 b$ & $489.6 \pm 26.0 c$ & $1082 \pm 37.0$ & $2.21 \pm 0.08 b$ & $1.90 \pm 0.08 c$ \\
\hline
\end{tabular}

Note. $\mathrm{T} 0=$ control 1 (without vaccination and without supplementation of $N$. sativa in diet), $\mathrm{T} 1=$ control $2(\mathrm{~T} 0+$ vaccination), $\mathrm{T} 2=\mathrm{T} 1+N$. sativa $0.5 \%$, $\mathrm{T} 3=\mathrm{T} 1+N$. sativa $1 \%, \mathrm{~T} 4=\mathrm{T} 1+N$. sativa $2 \%, \mathrm{~T} 5=\mathrm{T} 1+N$. sativa $4 \%, \mathrm{~T} 6=\mathrm{T} 1+N$. sativa $8 \%$, and $\mathrm{T} 7=\mathrm{T} 1+N$. sativa $16 \%$. Different superscript letters in each column/week indicate significant $(p<0.05)$ differences between groups, and NS stands for not significant $(p>0.05)$.

age, indicating that vaccination and $N$. sativa supplementation in feed did not strongly affect the MDA reduction rate of the chickens. (2) A significant difference $(p<0.05)$ between the unvaccinated and vaccinated groups on day 21 of age to the end of the experiment may indicate the effectiveness of the vaccination, while nearly undetectable antibody titers of unvaccinated chickens may confirm that there was no environmental or cross-contamination during the experimental period. (3) The lack of significant differences among the vaccinated groups on days 21,28 , and 35 of age may indicate that supplementation of different levels of $N$. sativa in diet affected the immune responses but did not have a strong effect on antibody titers of the treated groups, at least in a short period. Comparison antibody titers of the 


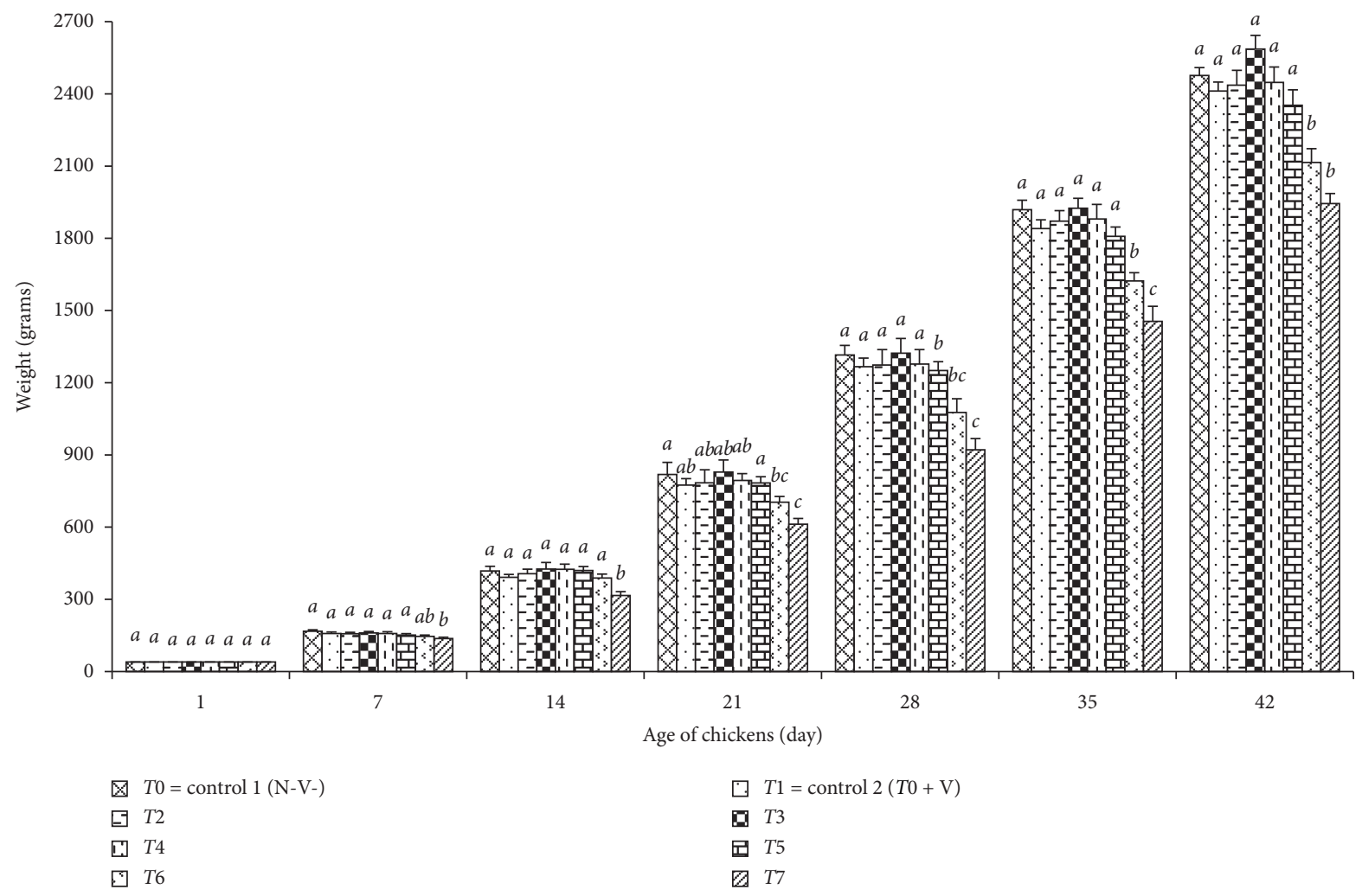

FIGURE 1: Effects of N. sativa on the weight of broilers: T0 = control 1 (without vaccination and without supplementation of $N$. sativa in diet), $\mathrm{T} 1=$ control $2(\mathrm{~T} 0+$ vaccination $), \mathrm{T} 2=\mathrm{T} 1+N$. sativa $0.5 \%, \mathrm{~T} 3=\mathrm{T} 1+N$. sativa $1 \%, \mathrm{~T} 4=\mathrm{T} 1+N$. sativa $2 \%, \mathrm{~T} 5=\mathrm{T} 1+N$. sativa $4 \%$, $\mathrm{T} 6=\mathrm{T} 1+N$. sativa $8 \%$, and $\mathrm{T} 7=\mathrm{T} 1+N$. sativa $16 \%$. Different alphabetical letters indicate significant $(p<0.05)$ differences between the means of the groups.

vaccinated groups at the end (day 42) of the experiment showed that antibody titers of group T3 (vaccinated and feed with a diet containing $1 \% N$. sativa seeds) differed significantly ( $p=0.027$ ) from that of group T1 (vaccinated but feed without $N$. sativa seeds diet) and from those of group T6 (vaccinated and feed with diet containing $8 \% \mathrm{~N}$. sativa seeds) and group T7 (vaccinated and feed with the diet containing $16 \% N$. sativa seeds) significantly ( $p=0.02$ and $p=0.01$, respectively) indicating effectiveness of different levels of $N$. sativa seeds supplementation in diets, as dietary inclusion of up to $1 \% N$. sativa seeds had the best immunomodulatory effects on the level of antibody titer following vaccination.

3.3. Hemogram. As shown in Table $3, \mathrm{RBC}$ and PCV as well as $\mathrm{Hb}$ values are age-dependent, and their values increased as the chickens get older. Weekly comparison of these parameters showed that supplementation of $N$. sativa seeds in the diet increases the values of RBC, PCV, and $\mathrm{Hb}$, and the group of chickens fed with $16 \%$ supplemented $N$. sativa seeds had the highest values and significantly $(p<0.05)$ differed from those of control groups (T0 and T1) as well as those of the treated groups (T2-T4) indicating that increasing effects of $N$. sativa seeds on hemogram parameters are dose-dependent (Table 3).
3.4. Leukogram. As shown in Table 4, WBC count of the vaccinated control 2 group $(\mathrm{T} 1=\mathrm{V}+\mathrm{N}-)$ was higher $(p>0.05)$ than that of the control 1 group $(\mathrm{T} 0=\mathrm{V}-\mathrm{N}-)$ from day 14 up to the end of the experiment indicating that WBC counts were affected by ND vaccination. Supplementation of $N$. sativa seeds affects leukogram parameters by decreasing WBC counts and lymphocytes and increasing heterophils, $H / L$ ratio, monocytes, eosinophils, and basophils percentages from day 21 of age until the end of the experiment. Weekly comparison (Table 4) of the groups indicates that the group (T7) fed with $16 \% N$. sativa seeds had the lowest WBC counts and lymphocyte percentages and their differences with control 2 group $(\mathrm{T} 1=\mathrm{T} 0+\mathrm{V})$ were significant $(p=0.013$ and $p=0.008$, respectively). But, values for $H / L$ ratio and percentages of heterophils, monocytes, eosinophils, and basophils of the group fed (T7) with $16 \% N$. sativa seeds were the highest and their values differed from those of the control 2 group $(\mathrm{T} 1=\mathrm{T} 0+\mathrm{V})$ significantly $(p=0.001)$.

\subsection{Biochemical Parameters}

3.5.1. Albumin and Total Protein. The concentration of albumin and total protein of control chickens increased during 6 weeks of the experimental period indicating that 


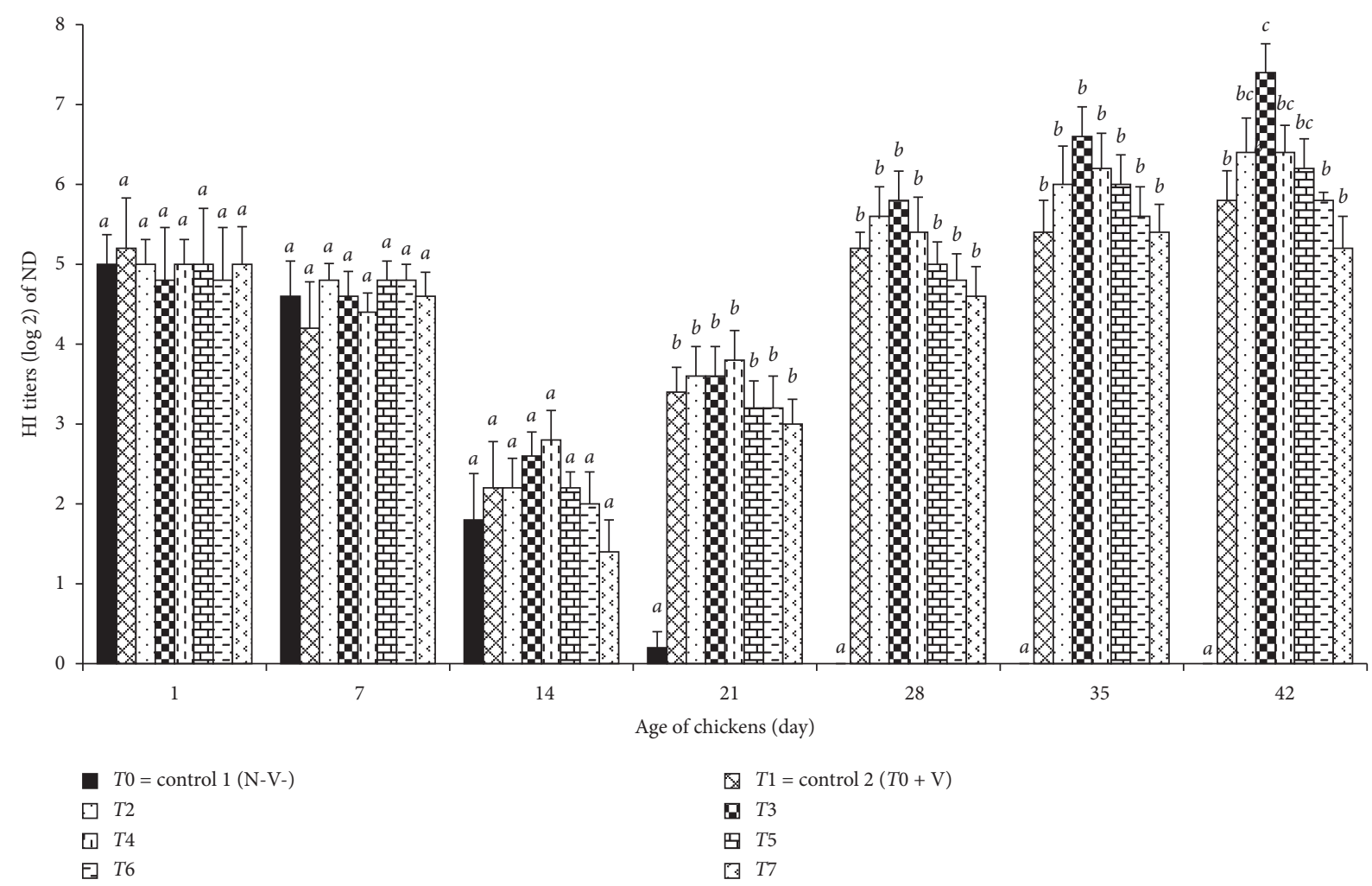

FIGURE 2: Effects of $N$. sativa on ND antibody titer of broilers: T0 = control 1 (without vaccination and without supplementation of $N$. sativa in diet), $\mathrm{T} 1=$ control $2(\mathrm{~T} 0+$ vaccination $), \mathrm{T} 2=\mathrm{T} 1+N$. sativa $0.5 \%, \mathrm{~T} 3=\mathrm{T} 1+N$. sativa $1 \%, \mathrm{~T} 4=\mathrm{T} 1+N$. sativa $2 \%, \mathrm{~T} 5=\mathrm{T} 1+N$. sativa $4 \%$, $\mathrm{T} 6=\mathrm{T} 1+N$. sativa $8 \%$, and $\mathrm{T} 7=\mathrm{T} 1+N$. sativa $16 \%$. Different alphabetical letters indicate significant $(p<0.05)$ differences between the means of the groups.

TABLE 3: Effects of $N$. sativa on hemogram (mean \pm SEM) of broiler chickens.

\begin{tabular}{|c|c|c|c|c|}
\hline Age (day) & Group & RBC $(\times 106)$ & PCV (\%) & $\mathrm{Hb}(\mathrm{g} / \mathrm{dl})$ \\
\hline \multirow[t]{4}{*}{ Day1 } & T0-T7 & $4.34 \pm 0.029 \mathrm{NS}$ & $24.2 \pm 0.00 \mathrm{NS}$ & $9.68 \pm 0.047 \mathrm{NS}$ \\
\hline & T0 & $5.00 \pm 0.054 \mathrm{a}$ & $27.2 \pm 0.00 \mathrm{a}$ & $10.98 \pm 0.015 a$ \\
\hline & $\mathrm{T} 1$ & $5.44 \pm 0.024 a$ & $31.0 \pm 0.00 \mathrm{a}$ & $11.40 \pm 0.044 \mathrm{a}$ \\
\hline & $\mathrm{T} 2$ & $5.5 \pm 0.024 a$ & $31.8 \pm 0.00 \mathrm{a}$ & $11.64 \pm 0.024 a$ \\
\hline \multirow{8}{*}{ Day 7} & $\mathrm{~T} 3$ & $5.54 \pm 0.024 a$ & $31.6 \pm 0.00 \mathrm{a}$ & $11.60 \pm 0.044 a$ \\
\hline & $\mathrm{T} 4$ & $5.52 \pm 0.040 \mathrm{a}$ & $30.6 \pm 0.00 \mathrm{a}$ & $11.20 \pm 0.063 \mathrm{a}$ \\
\hline & $\mathrm{T} 5$ & $5.50 \pm 0.019 a$ & $29.8 \pm 0.00 \mathrm{a}$ & $11.08 \pm 0.051 \mathrm{a}$ \\
\hline & T6 & $5.46 \pm 0.015 a$ & $29.3 \pm 0.00 \mathrm{a}$ & $10.92 \pm 0.020 \mathrm{a}$ \\
\hline & T7 & $5.42 \pm 0.048 \mathrm{a}$ & $29.0 \pm 0.00 \mathrm{a}$ & $10.78 \pm 0.020 \mathrm{a}$ \\
\hline & T0 & $4.32 \pm 0.048 a$ & $26.6 \pm 0.00 \mathrm{a}$ & $8.28 \pm 0.074 a$ \\
\hline & $\mathrm{T} 1$ & $4.34 \pm 0.060 \mathrm{a}$ & $26.8 \pm 0.00 \mathrm{ab}$ & $8.36 \pm 0.021 \mathrm{ab}$ \\
\hline & $\mathrm{T} 2$ & $4.44 \pm 0.024 a$ & $27.4 \pm 0.00 \mathrm{a}$ & $8.56 \pm 0.067 \mathrm{ab}$ \\
\hline \multirow{8}{*}{ Day 14} & T3 & $4.48 \pm 0.020 \mathrm{abc}$ & $27.8 \pm 0.00 \mathrm{~b}$ & $8.70 \pm 0.080 b$ \\
\hline & $\mathrm{T} 4$ & $4.50 \pm 0.067 b$ & $27.8 \pm 0.00 \mathrm{~b}$ & $8.72 \pm 0.019 b$ \\
\hline & T5 & $4.54 \pm 0.064 \mathrm{bcd}$ & $28.0 \pm 0.00 \mathrm{bc}$ & $8.76 \pm 0.015 b c$ \\
\hline & T6 & $4.62 \pm 0.073 \mathrm{cb}$ & $28.6 \pm 0.00 \mathrm{cbd}$ & $8.96 \pm 0.018 b c$ \\
\hline & $\mathrm{T} 7$ & $4.76 \mathrm{c} \pm 0.024 \mathrm{~d}$ & $29.6 \pm 0.01 \mathrm{~d}$ & $9.28 \pm 0.073 d$ \\
\hline & T0 & $4.62 \pm 0.048 \mathrm{a}$ & $28.6 \pm 0.00 \mathrm{a}$ & $8.98 \pm 0.073 a$ \\
\hline & $\mathrm{T} 1$ & $4.64 \pm 0.060 \mathrm{a}$ & $28.8 \pm 0.00 \mathrm{a}$ & $9.04 \pm 0.019 \mathrm{ab}$ \\
\hline & $\mathrm{T} 2$ & $4.74 \pm 0.024 \mathrm{ab}$ & $29.4 \pm 0.00 \mathrm{ba}$ & $9.22 \pm 0.073 a$ \\
\hline \multirow{5}{*}{ Day 21} & T3 & $4.78 \pm 0.020 \mathrm{ac}$ & $29.8 \pm 0.00 \mathrm{~b}$ & $9.34 \pm 0.060 \mathrm{bcde}$ \\
\hline & $\mathrm{T} 4$ & $4.80 \pm 0.067 \mathrm{bcd}$ & $29.8 \pm 0.00 b c$ & $9.34 \pm 0.018 c$ \\
\hline & T5 & $4.84 \pm 0.087 b c$ & $30.0 \pm 0.00 \mathrm{bcd}$ & $9.4 \pm 0.016 \mathrm{bcd}$ \\
\hline & T6 & $4.94 \pm 0.048 \mathrm{cb}$ & $30.6 \pm 0.00 \mathrm{cbd}$ & $9.58 \pm 0.014 \mathrm{dce}$ \\
\hline & $\mathrm{T} 7$ & $5.12 \pm 0.031 \mathrm{~d}$ & $31.6 \pm 0.00 \mathrm{~d}$ & $9.88 \pm 0.073 \mathrm{e}$ \\
\hline
\end{tabular}


TABLE 3: Continued.

\begin{tabular}{|c|c|c|c|c|}
\hline Age (day) & Group & $\mathrm{RBC}(\times 106)$ & PCV (\%) & $\mathrm{Hb}(\mathrm{g} / \mathrm{dl})$ \\
\hline \multirow{8}{*}{ Day 28} & T0 & $4.78 \pm 0.024 a$ & $29.6 \pm 0.00 \mathrm{a}$ & $9.28 \pm 0.073 a$ \\
\hline & $\mathrm{T} 1$ & $4.80 \pm 0.054 \mathrm{ab}$ & $29.8 \pm 0.00 \mathrm{ab}$ & $9.30 \pm 0.008 \mathrm{ab}$ \\
\hline & $\mathrm{T} 2$ & $4.88 \pm 0.048 \mathrm{ab}$ & $30.4 \pm 0.00 \mathrm{abc}$ & $9.52 \pm 0.073 \mathrm{abc}$ \\
\hline & $\mathrm{T} 3$ & $4.96 \pm 0.040 \mathrm{ac}$ & $30.8 \pm 0.00 \mathrm{bc}$ & $9.64 \pm 0.060 b c$ \\
\hline & $\mathrm{T} 4$ & $4.98 \pm 0.059 b c$ & $30.8 \pm 0.00 \mathrm{~cd}$ & $9.64 \pm 0.009 c d$ \\
\hline & T5 & $5.00 \pm 0.001 b c$ & $31.0 \pm 0.00 \mathrm{bcd}$ & $9.7 \pm 0.007 \mathrm{bcd}$ \\
\hline & T6 & $5.10 \pm 0.057 c d$ & $31.8 \pm 0.00$ dce & $9.88 \pm 0.008 \mathrm{dce}$ \\
\hline & $\mathrm{T} 7$ & $5.26 \pm 0.024 \mathrm{db}$ & $32.6 \pm 0.00 \mathrm{edc}$ & $10.18 \pm 0.073 \mathrm{edc}$ \\
\hline \multirow{8}{*}{ Day 35} & T0 & $5.12 \pm 0.048 \mathrm{a}$ & $31.6 \pm 0.00 \mathrm{a}$ & $9.88 \pm 0.073 a$ \\
\hline & $\mathrm{T} 1$ & $5.14 \pm 0.060 a$ & $31.8 \pm 0.00 \mathrm{ab}$ & $9.94 \pm 0.000 \mathrm{ab}$ \\
\hline & $\mathrm{T} 2$ & $5.24 \pm 0.024 \mathrm{ab}$ & $32.4 \pm 0.00 \mathrm{abc}$ & $10.12 \pm 0.073 \mathrm{ab}$ \\
\hline & T3 & $5.28 \pm 0.020 \mathrm{abc}$ & $32.8 \pm 0.00 \mathrm{bcd}$ & $10.24 \pm 0.060 \mathrm{bcc}$ \\
\hline & $\mathrm{T} 4$ & $5.30 \pm 0.067 \mathrm{bc}$ & $33.0 \pm 0.00 \mathrm{~cd}$ & $10.24 \pm 0.008 \mathrm{c}$ \\
\hline & $\mathrm{T} 5$ & $5.34 \pm 0.074 \mathrm{bcd}$ & $33.0 \pm 0.00 \mathrm{cbd}$ & $10.30 \pm 0.007 c$ \\
\hline & T6 & $5.42 \pm 0.073 \mathrm{~cd}$ & $33.6 \pm 0.00 \mathrm{dc}$ & $10.48 \pm 0.009 \mathrm{dce}$ \\
\hline & $\mathrm{T} 7$ & $5.56 \pm 0.024 \mathrm{dc}$ & $34.6 \pm 0.00 \mathrm{ed}$ & $10.78 \pm 0.073 \mathrm{ed}$ \\
\hline \multirow{8}{*}{ Day 42} & T0 & $5.56 \pm 0.024 a$ & $34.6 \pm 0.01 \mathrm{a}$ & $10.75 \pm 0.07 \mathrm{a}$ \\
\hline & $\mathrm{T} 1$ & $5.60 \pm 0.054 \mathrm{abc}$ & $34.8 \pm 0.01 \mathrm{ab}$ & $10.86 \pm 0.01 \mathrm{abc}$ \\
\hline & $\mathrm{T} 2$ & $5.68 \pm 0.048 \mathrm{ab}$ & $35.4 \pm 0.01 \mathrm{ab}$ & $11.06 \pm 0.09 \mathrm{abc}$ \\
\hline & T3 & $5.76 \pm 0.040 \mathrm{abcd}$ & $35.8 \pm 0.01 \mathrm{bcd}$ & $11.20 \pm 0.08 b c$ \\
\hline & $\mathrm{T} 4$ & $5.78 \pm 0.080 \mathrm{bc}$ & $35.8 \pm 0.01 b c$ & $11.22 \pm 0.01 b c$ \\
\hline & $\mathrm{T} 5$ & $5.80 \pm 0.010 b c$ & $36.0 \pm 0.01 b c$ & $11.26 \pm 0.01 \mathrm{bcd}$ \\
\hline & T6 & $5.90 \pm 0.080 \mathrm{~cd}$ & $36.6 \pm 0.01 \mathrm{~cd}$ & $11.46 \pm 0.01 \mathrm{bcd}$ \\
\hline & $\mathrm{T} 7$ & $6.06 \pm 0.024 d$ & $37.6 \pm 0.01 \mathrm{dc}$ & $11.78 \pm 0.07 \mathrm{~d}$ \\
\hline
\end{tabular}

Note. RBCs: red blood cells, PCV: packed cell volume, and Hb: hemoglobin. T0 $=$ control 1 (without vaccination and without supplementation of $N$. sativa in diet), $\mathrm{T} 1=\operatorname{control} 2(\mathrm{~T} 0+$ vaccination $), \mathrm{T} 2=\mathrm{T} 1+N$ sativa $0.5 \%, \mathrm{~T} 3=\mathrm{T} 1+N$ sativa $1 \%, \mathrm{~T} 4=\mathrm{T} 1+N$. sativa $2 \%, \mathrm{~T} 5=\mathrm{T} 1+N$. sativa $4 \%, \mathrm{~T} 6=\mathrm{T} 1+N$. sativa $8 \%$, and $\mathrm{T} 7=\mathrm{T} 1+N$. sativa $16 \%$. Different superscript letters in each column/week indicate significant $(p<0.05)$ differences between groups, and NS stands for not significant $(p>0.05)$.

TABLE 4: Effects of $N$. sativa on leukogram (mean \pm SEM) of broiler chickens.

\begin{tabular}{|c|c|c|c|c|c|c|c|c|}
\hline Age & Group & WBC $(\times 103)$ & Het $(\%)$ & Lym (\%) & Het/Lymp & Mon (\%) & Eos $(\%)$ & Bas (\%) \\
\hline \multirow[t]{4}{*}{ Day1 } & T0-T7 & $20.37 \pm 0.18 \mathrm{NS}$ & $37 \pm 7 \mathrm{NS}$ & $51.4 \pm 7 \mathrm{NS}$ & $0.94 \pm 0 \mathrm{NS}$ & $4 \pm 0 \mathrm{NS}$ & $6 \pm 0 \mathrm{NS}$ & $1.62 \pm 0 \mathrm{NS}$ \\
\hline & T0 & $29.1 \pm 0.10 \mathrm{a}$ & $30.0 \pm 1 \mathrm{a}$ & $55.0 \pm 0.8 \mathrm{a}$ & $0.54 \pm 0.00 \mathrm{a}$ & $4.8 \pm 0.0 \mathrm{acd}$ & $4.2 \pm 0 \mathrm{ac}$ & $6.02 \pm 0 \mathrm{a}$ \\
\hline & $\mathrm{T} 1$ & $29.2 \pm 0.20 \mathrm{a}$ & $38.8 \pm 1 b$ & $51.0 \pm 1 b$ & $0.76 \pm 0.00 \mathrm{~b}$ & $3.4 \pm 0.0 \mathrm{~b}$ & $3.22 \pm 0 b$ & $3.62 \pm 0 b$ \\
\hline & $\mathrm{T} 2$ & $28.6 \pm 0.21 \mathrm{a}$ & $37.2 \pm 1 b$ & $51.4 \pm 0.5 \mathrm{ab}$ & $0.72 \pm 0.02 b$ & $4.0 \pm 0.0 \mathrm{ab}$ & $3.42 \pm 0 \mathrm{ab}$ & $4.02 \pm 0 \mathrm{~b}$ \\
\hline \multirow{8}{*}{ Day 7} & T3 & $26.8 \pm 0.12 b$ & $34.6 \pm 1 \mathrm{ba}$ & $52.8 \pm 0.7 \mathrm{ab}$ & $0.65 \pm 0.02 b$ & $4.2 \pm 0.0 \mathrm{abc}$ & $4.02 \pm 0 \mathrm{abc}$ & $4.42 \pm 0 \mathrm{bc}$ \\
\hline & $\mathrm{T} 4$ & $25.0 \pm 0.41 b$ & $31.2 \pm 3 \mathrm{ca}$ & $54.8 \pm 0.0 \mathrm{a}$ & $0.56 \pm 0.03 a$ & $4.6 \pm 0.0 \mathrm{abc}$ & $4.42 \pm 0 c$ & $5.02 \pm 1 c d$ \\
\hline & T5 & $24.2 \pm 0.53 b$ & $27.2 \pm 1 \mathrm{cad}$ & $56.6 \pm 1 \mathrm{ca}$ & $0.48 \pm 0.09 a$ & $5.0 \pm 0.0 \mathrm{c}$ & $4.62 \pm 0 \mathrm{~cd}$ & $5.62 \pm 0 \mathrm{da}$ \\
\hline & T6 & $23.0 \pm 0.10 \mathrm{~cd}$ & $23.0 \pm 2 d$ & $60.2 \pm 1 d c$ & $0.38 \pm 0.01 \mathrm{c}$ & $5.4 \pm 0.0 \mathrm{~d}$ & $5.62 \pm 0 \mathrm{de}$ & $5.82 \pm 1 \mathrm{da}$ \\
\hline & T7 & $21.0 \pm 0.10 \mathrm{~d}$ & $21.2 \pm 4 \mathrm{~d}$ & $60.6 \pm 2 d$ & $0.34 \pm 0.00 c$ & $6.4 \pm 0.0 \mathrm{e}$ & $6.02 \pm 0 \mathrm{e}$ & $5.82 \pm 0 \mathrm{da}$ \\
\hline & T0 & $20.72 \pm 0.48 \mathrm{ab}$ & $47.0 \pm 1 \mathrm{a}$ & $46.0 \pm 0.1 \mathrm{a}$ & $1.02 \pm 0.02 \mathrm{a}$ & $3.7 \pm 0 \mathrm{a}$ & $2.2 \pm 0 \mathrm{a}$ & $1.62 \pm 0 \mathrm{a}$ \\
\hline & $\mathrm{T} 1$ & $21.40 \pm 0.50 \mathrm{a}$ & $45.8 \pm 1 \mathrm{a}$ & $45.8 \pm 0.3 \mathrm{a}$ & $1.00 \pm 0.04 \mathrm{a}$ & $3.8 \pm 0 \mathrm{a}$ & $2.82 \pm 0 \mathrm{a}$ & $1.82 \pm 0 \mathrm{a}$ \\
\hline & $\mathrm{T} 2$ & $19.36 \pm 0.72 \mathrm{ab}$ & $43.2 \pm 1 \mathrm{ab}$ & $46.6 \pm 0.5 a$ & $0.93 \pm 0.02 \mathrm{abc}$ & $4.4 \pm 0 \mathrm{ab}$ & $3.42 \pm 0 \mathrm{ab}$ & $2.42 \pm 0 \mathrm{ab}$ \\
\hline \multirow{8}{*}{ Day 14} & T3 & $18.82 \pm 0.86 \mathrm{bc}$ & $42.0 \pm 1 \mathrm{ba}$ & $46.6 \pm 0.7 \mathrm{a}$ & $0.89 \pm 0.02 b$ & $4.82 \pm 0 \mathrm{bc}$ & $3.82 \pm 0 \mathrm{bc}$ & $2.82 \pm 0 \mathrm{bc}$ \\
\hline & $\mathrm{T} 4$ & $18.43 \pm 1.56 b c$ & $41.8 \pm 3 b c$ & $46.6 \pm 0.0 \mathrm{a}$ & $0.89 \pm 0.04 b c$ & $4.92 \pm 0 \mathrm{bc}$ & $3.82 \pm 0 \mathrm{bc}$ & $2.92 \pm 0 \mathrm{bc}$ \\
\hline & T5 & $17.57 \pm 0.74 c$ & $41.4 \pm 1 b c$ & $46.6 \pm 0.6 \mathrm{a}$ & $0.88 \pm 0.06 b c$ & $5.02 \pm 0 b c$ & $4.02 \pm 0 b c$ & $3.02 \pm 0 b c$ \\
\hline & T6 & $17.18 \pm 1.24 c$ & $39.0 \pm 2 \mathrm{~cd}$ & $47.2 \pm 0.5 b$ & $0.82 \pm 0.05 c d$ & $5.62 \pm 0 \mathrm{~cd}$ & $4.62 \pm 0 \mathrm{~cd}$ & $3.62 \pm 0 \mathrm{~cd}$ \\
\hline & $\mathrm{T} 7$ & $16.90 \pm 0.58 c$ & $34.6 \pm 4 \mathrm{~d}$ & $48.6 \pm 0.8 b$ & $0.71 \pm 0.02 \mathrm{~d}$ & $6.62 \pm 0 \mathrm{~d}$ & $5.62 \pm 0 \mathrm{~d}$ & $4.62 \pm 0 \mathrm{~d}$ \\
\hline & T0 & $22.70 \pm 0.04 \mathrm{ab}$ & $33.6 \pm 0 \mathrm{a}$ & $52.6 \pm 0 \mathrm{a}$ & $0.64 \pm 0.01 \mathrm{a}$ & $4.62 \pm 0 \mathrm{a}$ & $6.62 \pm 0 \mathrm{a}$ & $2.62 \pm 0 \mathrm{a}$ \\
\hline & $\mathrm{T} 1$ & $22.95 \pm 0.05 a$ & $33.8 \pm 0 \mathrm{a}$ & $51.8 \pm 1 \mathrm{a}$ & $0.65 \pm 0.02 \mathrm{a}$ & $4.82 \pm 0 \mathrm{ab}$ & $6.82 \pm 0 \mathrm{ab}$ & $2.82 \pm 0 \mathrm{ab}$ \\
\hline & $\mathrm{T} 2$ & $20.91 \pm 0.07 \mathrm{abc}$ & $34.4 \pm 0 \mathrm{ab}$ & $49.4 \pm 0 \mathrm{ab}$ & $0.69 \pm 0.02 a$ & $5.42 \pm 0 \mathrm{abc}$ & $7.42 \pm 0 \mathrm{abc}$ & $3.42 \pm 0 \mathrm{abc}$ \\
\hline \multirow{5}{*}{ Day 21} & $\mathrm{~T} 3$ & $20.37 \pm 0.08 \mathrm{bcd}$ & $34.6 \pm 0 \mathrm{bd}$ & $48.6 \pm 0 \mathrm{~b}$ & $0.71 \pm 0.01 \mathrm{ab}$ & $5.62 \pm 0 \mathrm{bc}$ & $7.62 \pm 0 b$ & $3.62 \pm 0 b c$ \\
\hline & $\mathrm{T} 4$ & $20.12 \pm 0.10 \mathrm{cbd}$ & $34.8 \pm 0 \mathrm{bd}$ & $47.8 \pm 1 \mathrm{bd}$ & $0.73 \pm 0.03 \mathrm{ab}$ & $5.82 \pm 0 \mathrm{~cd}$ & $7.82 \pm 0 \mathrm{~cd}$ & $3.82 \pm 0 \mathrm{~cd}$ \\
\hline & T5 & $19.98 \pm 0.07 \mathrm{cbd}$ & $35.0 \pm 0 \mathrm{cb}$ & $47.0 \pm 2 \mathrm{cb}$ & $0.74 \pm .04 \mathrm{ab}$ & $6.02 \pm 0 \mathrm{~cd}$ & $8.02 \pm 0 \mathrm{~cd}$ & $4.02 \pm 0 \mathrm{~cd}$ \\
\hline & T6 & $18.73 \pm 0.12 \mathrm{~cd}$ & $35.6 \pm 0 \mathrm{dc}$ & $44.6 \pm 2$ dce & $0.80 \pm 0.04 b c$ & $6.62 \pm 0 \mathrm{~d}$ & $8.62 \pm 0 d$ & $4.62 \pm 0 \mathrm{~d}$ \\
\hline & $\mathrm{T} 7$ & $18.45 \pm .05 \mathrm{~d}$ & $36.6 \pm 0 \mathrm{e}$ & $40.6 \pm 0 \mathrm{e}$ & $0.90 \pm 0.02 c$ & $7.6 \pm 0 \mathrm{e}$ & $9.62 \pm 0 \mathrm{e}$ & $5.62 \pm 0 \mathrm{e}$ \\
\hline
\end{tabular}


TABLE 4: Continued.

\begin{tabular}{|c|c|c|c|c|c|c|c|c|}
\hline Age & Group & WBC $(\times 103)$ & Het $(\%)$ & Lym (\%) & Het/Lymp & Mon (\%) & Eos (\%) & Bas (\%) \\
\hline \multirow{8}{*}{ Day 28} & T0 & $23.80 \pm 0.04 a$ & $37.6 \pm 0 \mathrm{a}$ & $45.6 \pm 0 \mathrm{a}$ & $0.82 \pm 0.02 a$ & $5.62 \pm 0 \mathrm{a}$ & $7.62 \pm 0 \mathrm{a}$ & $3.62 \pm 0 \mathrm{a}$ \\
\hline & $\mathrm{T} 1$ & $24.50 \pm 0.05 \mathrm{ab}$ & $37.8 \pm 0 \mathrm{a}$ & $44.8 \pm 1 \mathrm{a}$ & $0.84 \pm 0.03 a$ & $5.72 \pm 0 \mathrm{ab}$ & $7.92 \pm 0 \mathrm{a}$ & $3.82 \pm 0 \mathrm{a}$ \\
\hline & $\mathrm{T} 2$ & $22.46 \pm 0.07 \mathrm{abc}$ & $38.4 \pm 0 \mathrm{ab}$ & $42.4 \pm 0 \mathrm{ab}$ & $0.90 \pm 0.02 \mathrm{ab}$ & $6.42 \pm 0 \mathrm{abc}$ & $8.32 \pm 0 \mathrm{ab}$ & $4.52 \pm 0 \mathrm{ab}$ \\
\hline & T3 & $21.90 \pm 0.08 \mathrm{abc}$ & $38.5 \pm 0 \mathrm{ab}$ & $41.6 \pm 0 b$ & $0.92 \pm 0.02 \mathrm{ab}$ & $6.62 \pm 0 \mathrm{bcd}$ & $8.52 \pm 0 \mathrm{ab}$ & $4.82 \pm 0 \mathrm{bc}$ \\
\hline & $\mathrm{T} 4$ & $21.67 \pm 0.01 \mathrm{ac}$ & $38.8 \pm 0 \mathrm{bc}$ & $40.6 \pm 2 b$ & $0.95 \pm 0.05 \mathrm{ab}$ & $6.82 \pm 0 \mathrm{~cd}$ & $8.92 \pm 0 \mathrm{bc}$ & $4.92 \pm 0 b c$ \\
\hline & T5 & $21.53 \pm 0.07 c$ & $39.0 \pm 0 b c$ & $40.0 \pm 2 b$ & $0.97 \pm 0.06 \mathrm{ab}$ & $7.02 \pm 0 \mathrm{dcd}$ & $9.02 \pm 0 b c$ & $5.02 \pm 0 b c$ \\
\hline & T6 & $20.28 \pm 0.12 \mathrm{dc}$ & $39.6 \pm 0 c$ & $37.6 \pm 2 \mathrm{~cd}$ & $1.05 \pm 0.07 b c$ & $7.52 \pm 0 \mathrm{~d}$ & $9.62 \pm 0 \mathrm{~cd}$ & $5.72 \pm 0 c$ \\
\hline & T7 & $20.00 \pm 0.05 \mathrm{dc}$ & $40.6 \pm 0 \mathrm{~d}$ & $33.6 \pm 0 \mathrm{~d}$ & $1.20 \pm 0.04 c$ & $8.52 \pm 0 \mathrm{e}$ & $10.42 \pm 0 \mathrm{~d}$ & $6.92 \pm 0 \mathrm{~d}$ \\
\hline \multirow{8}{*}{ Day 35} & T0 & $25.57 \pm 0.04 \mathrm{ab}$ & $35.4 \pm 0 \mathrm{a}$ & $57.8 \pm 0 \mathrm{a}$ & $0.61 \pm 0.01 \mathrm{a}$ & $3.62 \pm 0 \mathrm{a}$ & $2.52 \pm 0 \mathrm{a}$ & $0.72 \pm 0 \mathrm{a}$ \\
\hline & $\mathrm{T} 1$ & $26.25 \pm 0.05 a$ & $35.8 \pm 0 \mathrm{ab}$ & $56.8 \pm 1 \mathrm{ab}$ & $0.63 \pm 0.02 \mathrm{ab}$ & $3.92 \pm 0 \mathrm{ab}$ & $2.72 \pm 0 \mathrm{ab}$ & $0.82 \pm 0 \mathrm{ab}$ \\
\hline & $\mathrm{T} 2$ & $24.21 \pm 0.07 \mathrm{abc}$ & $36.3 \pm 0 \mathrm{ab}$ & $54.5 \pm 0 \mathrm{abc}$ & $0.66 \pm 0.01 \mathrm{ab}$ & $4.32 \pm 0 \mathrm{abc}$ & $3.52 \pm 0 \mathrm{abc}$ & $1.42 \pm 0 \mathrm{abc}$ \\
\hline & T3 & $23.67 \pm 0.08 \mathrm{abc}$ & $36.5 \pm 0 b c$ & $53.8 \pm 0 \mathrm{bc}$ & $0.68 \pm 0.01 \mathrm{ab}$ & $4.62 \pm 0 \mathrm{bc}$ & $3.52 \pm 0 \mathrm{bc}$ & $1.62 \pm 0 \mathrm{abc}$ \\
\hline & $\mathrm{T} 4$ & $23.42 \pm 0.10 b c$ & $36.8 \pm 0 c$ & $52.7 \pm 1 c$ & $0.70 \pm 0.03 \mathrm{abc}$ & $4.82 \pm 0 \mathrm{bcd}$ & $3.92 \pm 0 \mathrm{~cd}$ & $1.82 \pm 0 \mathrm{~cd}$ \\
\hline & T5 & $23.28 \pm 0.07 b c$ & $37.0 \pm 0 c$ & $52.0 \pm 2 c$ & $0.71 \pm 0.04 \mathrm{abc}$ & $5.02 \pm 0 \mathrm{~cd}$ & $4.02 \pm 0 \mathrm{~cd}$ & $2.02 \pm 0 \mathrm{~cd}$ \\
\hline & T6 & $22.03 \pm 0.12 c$ & $37.6 \pm 0 \mathrm{~d}$ & $49.7 \pm 2 d$ & $0.76 \pm 0.04 b c$ & $5.52 \pm 0 \mathrm{~d}$ & $4.62 \pm 0 \mathrm{~d}$ & $2.62 \pm 0 \mathrm{de}$ \\
\hline & T7 & $21.72 \pm 0.05 c$ & $38.6 \pm 0 \mathrm{e}$ & $45.7 \pm 0 \mathrm{e}$ & $0.84 \pm 0.02 c$ & $6.62 \pm 0 \mathrm{e}$ & $5.62 \pm 0 \mathrm{e}$ & $3.52 \pm 0 \mathrm{e}$ \\
\hline \multirow{8}{*}{ Day 42} & T0 & $27.12 \pm 0.04 \mathrm{ab}$ & $33.6 \pm \pm 0 \mathrm{a}$ & $54.7 \pm 0 \mathrm{a}$ & $0.61 \pm 0.01 \mathrm{a}$ & $3.62 \pm 0 \mathrm{a}$ & $5.52 \pm 0 \mathrm{a}$ & $2.62 \pm 0 \mathrm{a}$ \\
\hline & $\mathrm{T} 1$ & $27.80 \pm 0.05 a$ & $33.8 \pm \pm 0 \mathrm{ab}$ & $53.9 \pm 1 \mathrm{ab}$ & $0.63 \pm 0.02 \mathrm{ab}$ & $3.82 \pm 0 \mathrm{ab}$ & $5.72 \pm 0 \mathrm{ab}$ & $2.82 \pm 0 \mathrm{ab}$ \\
\hline & $\mathrm{T} 2$ & $25.76 \pm 0.07 \mathrm{abc}$ & $34.4 \pm \pm 0 \mathrm{abc}$ & $51.5 \pm 0 \mathrm{abc}$ & $0.66 \pm 0.01 \mathrm{ab}$ & $4.32 \pm 0 \mathrm{abc}$ & $6.42 \pm 0 \mathrm{abc}$ & $3.42 \pm 0 \mathrm{abc}$ \\
\hline & $\mathrm{T} 3$ & $25.22 \pm 0.08 \mathrm{abc}$ & $34.6 \pm 0 \mathrm{bc}$ & $50.6 \pm 0 \mathrm{bc}$ & $0.68 \pm 0.01 \mathrm{abc}$ & $4.62 \pm 0 \mathrm{bcd}$ & $6.52 \pm 0 \mathrm{bc}$ & $3.72 \pm 0 \mathrm{bcd}$ \\
\hline & $\mathrm{T} 4$ & $24.83 \pm 0.10 b c$ & $34.9 \pm 0 \mathrm{~cd}$ & $49.7 \pm 1 b c d$ & $0.70 \pm 0.03 \mathrm{abc}$ & $4.82 \pm 0 \mathrm{~cd}$ & $6.82 \pm 0 \mathrm{~cd}$ & $3.82 \pm 0 \mathrm{~cd}$ \\
\hline & T5 & $24.67 \pm 0.07 b c$ & $35.0 \pm 0 \mathrm{~cd}$ & $49.0 \pm 2 \mathrm{~cd}$ & $0.71 \pm 0.04 b c$ & $5.02 \pm 0 \mathrm{~cd}$ & $7.02 \pm 0 \mathrm{~cd}$ & $4.02 \pm 0 \mathrm{~cd}$ \\
\hline & T6 & $23.58 \pm 0.12 c$ & $35.6 \pm 0 \mathrm{~d}$ & $46.7 \pm 2 d$ & $0.76 \pm 0.04 c$ & $5.52 \pm 0 \mathrm{~d}$ & $7.62 \pm 0 \mathrm{~d}$ & $4.62 \pm 0 \mathrm{~d}$ \\
\hline & $\mathrm{T} 7$ & $23.30 \pm 0.05 c$ & $36.6 \pm 0 \mathrm{e}$ & $42.5 \pm 0 \mathrm{e}$ & $0.86 \pm 0.02 \mathrm{~d}$ & $6.62 \pm 0 \mathrm{e}$ & $8.72 \pm 0 \mathrm{e}$ & $5.62 \pm 0 \mathrm{e}$ \\
\hline
\end{tabular}

Note. WBC: white blood cells, Het: heterophils, Lym: lymphocytes, H/L: heterophils to lymphocytes ratio, Mon: monocytes, Eos: eosinophils, and Bas: basophils. $\mathrm{T} 0=$ control 1 (without vaccination and without supplementation of $N$. sativa in diet), $\mathrm{T} 1=$ control $2(\mathrm{~T} 0+$ vaccination), $\mathrm{T} 2=\mathrm{T} 1+N$. sativa $0.5 \%$, $\mathrm{T} 3=\mathrm{T} 1+N$. sativa $1 \%, \mathrm{~T} 4=\mathrm{T} 1+N$. sativa $2 \%, \mathrm{~T} 5=\mathrm{T} 1+N$. sativa $4 \%, \mathrm{~T} 6=\mathrm{T} 1+N$. sativa $8 \%$, and $\mathrm{T} 7=\mathrm{T} 1+N$. sativa $16 \%$. Different superscript letters in each column/week indicate significant $(p<0.05)$ differences between groups, and NS stands for not significant $(p>0.05)$.

these parameters are age-dependent. A weekly comparison of the groups (Table 5) showed that supplementation of up to $2 \%$ of $N$. sativa seeds in the diet increases serum level of these parameters whereas $4 \%$ and over that decrease concentrations of these elements in the serum of broiler chickens. Overall, albumin and total protein levels of chickens fed with a diet containing different $N$. sativa seeds were higher than those of the control groups (T0-T1) and the differences were significant $(p<0.05)$ when compared with those of the control groups (T0-T1).

3.5.2. Glucose. As shown in Table 5, weekly comparison of the control groups (T0-T1) revealed that the serum glucose concentration of the chickens is decreased by age, and vaccination against ND did not affect its level. A weekly comparison of the treated groups indicated that the effects of $N$. sativa on serum glucose were dose-dependent. On the other hand, increasing the effect on serum glucose of the chickens was observed by 0.5 to $2 \%$ of $N$. sativa seed supplementation in diet, while serum glucose of the chickens fed with $4 \%$ and over that decreased gradually (Table 5). Overall, the chickens fed with different concentrations of $N$. sativa seeds in the feed had higher glucose level than those of the control groups (T0T1), and a weekly comparison of the serum glucose level of the groups showed that on age 14 up to the end of the experiment, chickens of the group (T4) fed with $2 \% N$. sativa diet had the highest level of serum glucose and differences between the groups were significant $(p<0.05)$.
3.5.3. Calcium and Phosphorus. As shown in Table 5, supplementation of up to $2 \%$ of $N$. sativa seeds in diet had increasing effects on levels of serum calcium and phosphorus, while $4 \%$ and over that had decreasing effects. Chickens fed with $2 \%$ of $N$. sativa seeds in the diet had the highest level of serum calcium and phosphorus that significantly $(p<0.05)$ differed from those of the control groups ( $\mathrm{T} 0$ and $\mathrm{T} 1)$.

3.5.4. Cholesterol and Triglyceride. As shown in Table 6, high levels of cholesterol and triglyceride due to yolk residue reduced sharply in the $1^{\text {st }}$ week, and then their levels reduced gradually during the experimental period, indicating that their concentrations are age-dependent at least in broilers up to 42 days of age. Weekly comparison of $N$. sativa treated and control groups showed that inclusion of N. sativa seeds in broiler's diets reduces the concentration of both these parameters, and chickens of group T7 fed with $16 \%$ N. sativa seeds had the lowest level and differed significantly $(p<0.05)$ from those of the control groups (T0-T1).

3.5.5. Serum Enzymes (ALP, ALT, and AST). As shown in Table 6, the lack of significant differences between group T0 and group $\mathrm{T} 1$ indicates that vaccination does not affect the serum enzyme levels, and levels of both ALP and AST were decreased by age, while those of ALT were increased. Weekly comparison (Table 6) of the groups revealed the following. (a) The levels of ALP decreased by the inclusion of N. sativa 
TABLE 5: Effects of N. sativa on biochemical parameters (Mean \pm SEM) of broilers.

\begin{tabular}{|c|c|c|c|c|c|c|}
\hline Age (day) & Group & Alb (g/dL) & $\mathrm{TP}(\mathrm{g} / \mathrm{dL})$ & Glu (mg/dL) & Cal (mg/dL) & Phos (mg/dL) \\
\hline \multirow[t]{4}{*}{ Dayl } & T0-T7 & $0.62 \pm 0.03 \mathrm{NS}$ & $1.73 \pm 0.10 \mathrm{NS}$ & $276 \pm 7.5 \mathrm{NS}$ & $8.19 \pm 0.07 \mathrm{NS}$ & $2.09 \pm 0.02 \mathrm{NS}$ \\
\hline & T0 & $0.74 \pm 0.05 a$ & $1.85 \pm 0.10 \mathrm{a}$ & $220.6 \pm 304 \mathrm{ad}$ & $8.0 \pm 0.03 \mathrm{a}$ & $3.03 \pm 0.04 a$ \\
\hline & $\mathrm{T} 1$ & $0.72 \pm 0.02 \mathrm{a}$ & $1.86 \pm 0.02 \mathrm{a}$ & $216.0 \pm 0.94 \mathrm{a}$ & $8.06 \pm 0.05 a$ & $30.8 \pm 0.12 \mathrm{a}$ \\
\hline & $\mathrm{T} 2$ & $0.76 \pm 0.02 a$ & $1.87 \pm 0.03 \mathrm{ab}$ & $225.0 \pm 2.56 \mathrm{abd}$ & $8.26 \pm 0.04 a$ & $3.52 \pm 0.06 b$ \\
\hline \multirow{8}{*}{ Day 7} & $\mathrm{~T} 3$ & $0.78 \pm 0.03 a$ & $2.06 \pm 0.06 b$ & $232.8 \pm 1.99 b c$ & $8.36 \pm 0.11 b$ & $3.66 \pm 0.08 \mathrm{bc}$ \\
\hline & $\mathrm{T} 4$ & $0.84 \pm 0.03 a$ & $2.06 \pm 0.10 \mathrm{~b}$ & $240.4 \pm 0.52 b$ & $9.60 \pm 0.05 c$ & $3.78 \pm 0.08 c$ \\
\hline & $\mathrm{T} 5$ & $0.80 \pm 0.05 \mathrm{a}$ & $1.96 \pm 0.06 \mathrm{~b}$ & $230.4 \pm 2.88 \mathrm{~cd}$ & $8.18 \pm 0.03 \mathrm{ab}$ & $3.74 \pm 0.04 \mathrm{cb}$ \\
\hline & T6 & $0.70 \pm 0.01 \mathrm{a}$ & $1.78 \pm 0.05 \mathrm{ab}$ & $227.0 \pm 1.86 \mathrm{db}$ & $7.52 \pm 0.04 \mathrm{ad}$ & $3.71 \pm 0.09 \mathrm{cb}$ \\
\hline & $\mathrm{T} 7$ & $0.68 \pm 0.03 a$ & $1.74 \pm 0.08 \mathrm{a}$ & $221.4 \pm 2.04 \mathrm{ad}$ & $7.25 \pm 0.05 \mathrm{~d}$ & $3.61 \pm 0.05 \mathrm{cb}$ \\
\hline & T0 & $0.74 \pm 0.07 a$ & $2.14 \pm 0.05 a$ & $221.8 \pm 1.93 \mathrm{ad}$ & $8.50 \pm 0.14 a$ & $3.26 \pm 0.08 \mathrm{a}$ \\
\hline & $\mathrm{T} 1$ & $0.68 \pm 0.07 a$ & $1.92 \pm 0.08 \mathrm{abc}$ & $217.6 \pm 2.18 \mathrm{a}$ & $8.48 \pm 0.16 a$ & $3.14 \pm 0.03 a$ \\
\hline & $\mathrm{T} 2$ & $0.70 \pm 0.05 a$ & $1.96 \pm 0.15 \mathrm{abc}$ & $239.4 \pm 1.24 b$ & $8.90 \pm 0.06 a$ & $3.40 \pm 0.06 b$ \\
\hline \multirow{8}{*}{ Day 14} & T3 & $0.72 \pm 0.05 a$ & $2.04 \pm 0.12 \mathrm{abc}$ & $246.8 \pm 3.39 b c$ & $9.06 \pm 0.04 a$ & $3.48 \pm 0.05 b$ \\
\hline & $\mathrm{T} 4$ & $0.80 \pm 0.0 \mathrm{a}$ & $2.26 \pm 0.14 \mathrm{a}$ & $254.6 \pm 1.03 c$ & $9.06 \pm 0.08 b$ & $3.86 \pm 0.07 c$ \\
\hline & T5 & $0.70 \pm 0.07 a$ & $1.90 \pm 0.14 \mathrm{abc}$ & $250.0 \pm 3.56 c$ & $7.44 \pm 0.12 c$ & $3.50 \pm 0.06 b$ \\
\hline & T6 & $0.68 \pm 0.06 a$ & $1.86 \pm 0.08 \mathrm{ab}$ & $244.2 \pm 3.42 b c$ & $7.24 \pm 0.16 c$ & $3.02 \pm 0.05 a$ \\
\hline & $\mathrm{T} 7$ & $0.64 \pm 0.04 a$ & $1.68 \pm 0.13 \mathrm{cb}$ & $227.4 \pm 3.50 \mathrm{~d}$ & $6.94 \pm 0.13 c$ & $3.10 \pm 0.04 a$ \\
\hline & T0 & $0.94 \pm 0.07 a$ & $2.34 \pm 0.08 \mathrm{a}$ & $220.8 \pm 1.9 \mathrm{ad}$ & $8.24 \pm 0.16 \mathrm{a}$ & $3.30 \pm 0.07 \mathrm{a}$ \\
\hline & $\mathrm{T} 1$ & $0.72 \pm 0.07 a$ & $2.08 \pm 0.10 \mathrm{abc}$ & $224.2 \pm 2.1 \mathrm{ad}$ & $8.18 \pm 0.16 b$ & $3.20 \pm 0.06 \mathrm{a}$ \\
\hline & $\mathrm{T} 2$ & $0.94 \pm 0.05 a$ & $2.12 \pm 0.17 \mathrm{abc}$ & $251.2 \pm 1.2 b$ & $9.50 \pm 0.06 b$ & $3.66 \pm 0.03 b$ \\
\hline \multirow{8}{*}{ Day 21} & $\mathrm{~T} 3$ & $0.98 \pm 0.05 a$ & $2.22 \pm 0.12 \mathrm{abc}$ & $269.0 \pm 2.3 c$ & $9.66 \pm 0.04 b$ & $3.80 \pm 0.02 c$ \\
\hline & $\mathrm{T} 4$ & $1.0 \pm 0.05 \mathrm{a}$ & $2.46 \pm 0.16 \mathrm{a}$ & $276.6 \pm 1.0 \mathrm{c}$ & $10.98 \pm 0.08 c$ & $3.92 \pm 0.03 c$ \\
\hline & T5 & $0.90 \pm 0.07 a$ & $2.06 \pm 0.15 a b c$ & $272.0 \pm 2.5 c$ & $7.96 \pm 0.14 a$ & $3.62 \pm 0.05 b$ \\
\hline & T6 & $0.88 \pm 0.06 a$ & $1.98 \pm 0.10 b$ & $269.2 \pm 2.4 c$ & $7.76 \pm 0.16 a$ & $3.14 \pm 0.08 \mathrm{ad}$ \\
\hline & $\mathrm{T} 7$ & $0.88 \pm 0.04 a$ & $1.80 \pm 0.15 \mathrm{cb}$ & $224.4 \pm 3.5 \mathrm{~d}$ & $7.47 \pm 0.13 a$ & $3.00 \pm 0.05 \mathrm{~d}$ \\
\hline & T0 & $1.34 \pm 0.07 \mathrm{a}$ & $2.53 \pm 0.10 \mathrm{a}$ & $209.8 \pm 1.9 \mathrm{a}$ & $8.44 \pm 0.16 \mathrm{a}$ & $3.38 \pm 0.05 a$ \\
\hline & $\mathrm{T} 1$ & $1.34 \pm 0.07 \mathrm{a}$ & $2.56 \pm 0.10 \mathrm{a}$ & $213.6 \pm 2.1 \mathrm{a}$ & $8.36 \pm 0.16 a$ & $3.30 \pm 0.08 \mathrm{a}$ \\
\hline & $\mathrm{T} 2$ & $1.60 \pm 0.05 b$ & $3.08 \pm 0.10 \mathrm{bcd}$ & $260.2 \pm 1.2 b$ & $9.80 \pm 0.08 b$ & $3.70 \pm 0.03 b$ \\
\hline \multirow{8}{*}{ Day 28} & $\mathrm{~T} 3$ & $1.62 \pm 0.05 b$ & $3.18 \pm 0.09 b c d$ & $278.0 \pm 2.3 c$ & $9.96 \pm 0.04 b$ & $3.90 \pm 0.02 c$ \\
\hline & $\mathrm{T} 4$ & $1.80 \pm 0.05 c$ & $3.40 \pm 0.10 \mathrm{~b}$ & $285.6 \pm 1.0 \mathrm{c}$ & $11.08 \pm 0.08 c$ & $4.02 \pm 0.03 c$ \\
\hline & $\mathrm{T} 5$ & $1.68 \pm 0.07 b c$ & $3.18 \pm 0.10 \mathrm{bcd}$ & $281.0 \pm 2.6 c$ & $8.26 \pm 0.14 \mathrm{ad}$ & $3.72 \pm 0.05 b$ \\
\hline & T6 & $1.68 \pm 0.06 b c$ & $3.04 \pm 0.10 \mathrm{~cd}$ & $278.2 \pm 2.4 c$ & $8.06 \pm 0.16 \mathrm{ad}$ & $3.24 \pm 0.08 \mathrm{ad}$ \\
\hline & $\mathrm{T} 7$ & $1.58 \pm 0.04 b$ & $3.00 \pm 0.08 \mathrm{dc}$ & $228.0 \pm 2.5 \mathrm{~d}$ & $7.76 \pm 0.13 \mathrm{~d}$ & $3.10 \pm 0.05 \mathrm{~d}$ \\
\hline & T0 & $1.54 \pm 0.07 \mathrm{a}$ & $2.94 \pm 0.12 \mathrm{a}$ & $203.8 \pm 1.93 a$ & $8.54 \pm 0.16 a$ & $3.40 \pm 0.07 \mathrm{a}$ \\
\hline & $\mathrm{T} 1$ & $1.54 \pm 0.07 \mathrm{a}$ & $2.94 \pm 0.12 \mathrm{a}$ & $211.6 \pm 2.18 \mathrm{a}$ & $8.68 \pm 0.16 a$ & $3.32 \pm 0.06 \mathrm{a}$ \\
\hline & $\mathrm{T} 2$ & $1.70 \pm 0.05 \mathrm{ab}$ & $3.24 \pm 0.11 \mathrm{abc}$ & $255.2 \pm 1.24 \mathrm{~b}$ & $9.90 \pm 0.06 b c$ & $3.76 \pm 0.03 b$ \\
\hline \multirow{8}{*}{ Day 35} & $\mathrm{~T} 3$ & $1.82 \pm 0.06 b$ & $3.36 \pm 0.11 b c$ & $273.0 \pm 3.39 c$ & $10.05 \pm 0.04 b c$ & $4.00 \pm 0.02 c$ \\
\hline & $\mathrm{T} 4$ & $1.88 \pm 0.05 b$ & $3.56 \pm 0.10 b$ & $280.6 \pm 1.02 c$ & $10.38 \pm 0.08 c$ & $4.04 \pm 0.04 c$ \\
\hline & T5 & $1.78 \pm 0.07 \mathrm{bc}$ & $3.44 \pm 0.12 \mathrm{bc}$ & $272.0 \pm 3.56 c$ & $8.36 \pm 0.14 a$ & $3.74 \pm 0.05 b$ \\
\hline & T6 & $1.72 \pm 0.06 \mathrm{ab}$ & $3.22 \pm 0.11 \mathrm{abc}$ & $259.2 \pm 3.42 \mathrm{db}$ & $8.16 \pm 0.16 \mathrm{ad}$ & $3.26 \pm 0.05 \mathrm{ad}$ \\
\hline & $\mathrm{T} 7$ & $1.68 \pm 0.06 \mathrm{ac}$ & $3.18 \pm 0.11 \mathrm{ca}$ & $204.4 \pm 3.50 \mathrm{ea}$ & $7.80 \pm 0.14 \mathrm{~d}$ & $3.14 \pm 0.06 \mathrm{~d}$ \\
\hline & T0 & $1.74 \pm 0.07 \mathrm{a}$ & $3.24 \pm 0.12 \mathrm{a}$ & $180.8 \pm 1.92 \mathrm{a}$ & $8.92 \pm 0.1 \mathrm{a}$ & $3.60 \pm 0.08 \mathrm{a}$ \\
\hline & $\mathrm{T} 1$ & $1.72 \pm 0.07 a$ & $3.22 \pm 0.12 \mathrm{a}$ & $194.6 \pm 2.17 b$ & $9.14 \pm 0.2 \mathrm{a}$ & $3.52 \pm 0.06 a$ \\
\hline & $\mathrm{T} 2$ & $1.92 \pm 0.06 \mathrm{abc}$ & $3.54 \pm 0.11 \mathrm{abc}$ & $232.2 \pm 3.38 c$ & $11.68 \pm 0.1 b$ & $3.96 \pm 0.04 b$ \\
\hline \multirow{5}{*}{ Day 42} & T3 & $2.02 \pm 0.05 b c$ & $3.66 \pm 0.10 b c$ & $250.0 \pm 1.12 \mathrm{~d}$ & $11.86 \pm 0.1 b$ & $4.20 \pm 0.03 c$ \\
\hline & $\mathrm{T} 4$ & $2.08 \pm 0.06 b$ & $3.86 \pm 0.12 b$ & $257.6 \pm 3.56 \mathrm{~d}$ & $12.06 \pm 0.1 b$ & $4.24 \pm 0.04 c$ \\
\hline & T5 & $1.98 \pm 0.07 b c$ & $3.74 \pm 0.11 b c$ & $243.6 \pm 3.42 \mathrm{~d}$ & $9.92 \pm 0.2 \mathrm{c}$ & $3.94 \pm 0.05 b$ \\
\hline & T6 & $1.90 \pm 0.06 \mathrm{abc}$ & $3.52 \pm 0.11 \mathrm{abc}$ & $238.2 \pm 2.50 c$ & $9.72 \pm 0.1 c$ & $3.46 \pm 0.06 \mathrm{a}$ \\
\hline & T7 & $1.85 \pm 0.05 \mathrm{ac}$ & $3.48 \pm 0.11 \mathrm{ac}$ & $198.0 \pm 1.12 b$ & $9.36 \pm 0.1 \mathrm{da}$ & $3.34 \pm 0.06 \mathrm{da}$ \\
\hline
\end{tabular}

Note. Alb: albumin, TP: total protein, Glu: glucose, Cal: calcium, and Ph: phosphorous. T0 = control 1 (without vaccination and without supplementation of $N$ sativa in diet), $\mathrm{T} 1=$ control $2(\mathrm{~T} 0+$ vaccination $), \mathrm{T} 2=\mathrm{T} 1+N$. sativa $0.5 \%, \mathrm{~T} 3=\mathrm{T} 1+N$. sativa $1 \%, \mathrm{~T} 4=\mathrm{T} 1+N$. sativa $2 \%, \mathrm{~T} 5=\mathrm{T} 1+N$. sativa $4 \%$, $\mathrm{T} 6=\mathrm{T} 1+N$. sativa $8 \%$, and $\mathrm{T} 7=\mathrm{T} 1+N$. sativa $16 \%$. Different superscript letters in each column/week indicate significant $(p<0.05)$ differences between groups, and NS stands for not significant $(p>0.05)$.

seeds in the diet during the experimental period and group T7 had the lowest levels of ALP which significantly $(p<0.05)$ differed from those of control groups (T0-T1) from age 14 to 42 days. (b) Inclusion of up to $2 \%$ of N. sativa seeds in the diet increased the levels of ALT while $8 \%$ and over that decreased levels of ALT. Therefore, group T4 ( $2 \%$ N. sativa) had the highest level of ALT and group T7 (16\% N. sativa) had the lowest level of ALT. The mean differences between the control groups with groups T4 ( $2 \% N$. sativa) and T7 $(16 \% N$. sativa $)$ were significant $(p<0.05)$ on days 28,35 , and 42 of age). The level of AST is decreased gradually during the experimental period. Weekly comparison (Table 6) of the 
TABLE 6: Effects of N. sativa on biochemical parameters (Mean \pm SEM) of broilers.

\begin{tabular}{|c|c|c|c|c|c|c|}
\hline Age (day) & Group & Chol (mg/dL) & Trigl (mg/dL) & $\operatorname{ALP}(\mathrm{U} / \mathrm{L})$ & ALT (U/L) & AST (U/L) \\
\hline \multirow[t]{4}{*}{ Dayl } & T0-T7 & $304.9 \pm 6.7 \mathrm{NS}$ & $179.825 \pm 5.1 \mathrm{NS}$ & $12.03 \pm 0.17 \mathrm{NS}$ & $7.9 \pm 0.3 \mathrm{NS}$ & $218.4 \pm 2.6 \mathrm{NS}$ \\
\hline & T0 & $154.8 \pm 2.7 \mathrm{a}$ & $137.0 \pm 2.7 \mathrm{a}$ & $10.38 \pm 0.12 \mathrm{a}$ & $9.2 \pm 0.3 \mathrm{a}$ & $241.2 \pm 2.3 \mathrm{a}$ \\
\hline & $\mathrm{T} 1$ & $155.2 \pm 1.9 \mathrm{a}$ & $132.0 \pm 1.8 \mathrm{~b}$ & $10.28 \pm 0.22 \mathrm{a}$ & $9.6 \pm 0.4 \mathrm{a}$ & $246.8 \pm 2.6 \mathrm{a}$ \\
\hline & $\mathrm{T} 2$ & $156.2 \pm 1.6 \mathrm{a}$ & $115.8 \pm 2.0 \mathrm{c}$ & $10.03 \pm 0.13 \mathrm{a}$ & $9.8 \pm 0.3 \mathrm{a}$ & $237.6 \pm 2.4 b$ \\
\hline \multirow{8}{*}{ Day 7} & $\mathrm{~T} 3$ & $157.2 \pm 1.5 \mathrm{a}$ & $115.4 \pm 2.1 \mathrm{c}$ & $9.94 \pm 0.10 \mathrm{a}$ & $10.6 \pm 0.4 b$ & $236.8 \pm 2.5 b$ \\
\hline & $\mathrm{T} 4$ & $155.4 \pm 1.2 \mathrm{a}$ & $112.4 \pm 2.1 \mathrm{c}$ & $9.92 \pm 0.10 \mathrm{a}$ & $11.2 \pm 0.5 b$ & $233.8 \pm 2.8 b c$ \\
\hline & T5 & $155.8 \pm 1.5 \mathrm{a}$ & $107.2 \pm 1.9 \mathrm{~d}$ & $9.91 \pm 0.13 \mathrm{a}$ & $11.0 \pm 0.3 b$ & $228.4 \pm 2.4 \mathrm{~cd}$ \\
\hline & T6 & $154.0 \pm 1.7 \mathrm{a}$ & $105.8 \pm 2.0 \mathrm{~d}$ & $9.91 \pm 0.10 \mathrm{a}$ & $9.2 \pm 0.3 \mathrm{a}$ & $227.6 \pm 2.6 \mathrm{~d}$ \\
\hline & $\mathrm{T} 7$ & $154.6 \pm 1.3 \mathrm{a}$ & $103.4 \pm 2.4 \mathrm{~d}$ & $9.82 \pm 0.10 \mathrm{a}$ & $8.0 \pm 0.4 \mathrm{c}$ & $227.0 \pm 2.2 \mathrm{~d}$ \\
\hline & T0 & $154.4 \pm 1.3 \mathrm{a}$ & $173.2 \pm 3.4 \mathrm{a}$ & $8.96 \pm 0.14 a$ & $9.7 \pm 0.4 \mathrm{ac}$ & $234.6 \pm 2.4 \mathrm{a}$ \\
\hline & $\mathrm{T} 1$ & $150.4 \pm 2.4 a$ & $172.0 \pm 2.7 \mathrm{a}$ & $8.98 \pm 0.20 a$ & $9.6 \pm 0.3 \mathrm{ac}$ & $234.0 \pm 2.5 \mathrm{a}$ \\
\hline & $\mathrm{T} 2$ & $151.6 \pm 1.7 \mathrm{a}$ & $167.4 \pm 3.1 \mathrm{~b}$ & $8.78 \pm 0.10 \mathrm{ab}$ & $10.0 \pm 0.5 \mathrm{a}$ & $230.0 \pm 2.5 a$ \\
\hline \multirow{8}{*}{ Day 14} & $\mathrm{~T} 3$ & $150.4 \pm 1.5 \mathrm{a}$ & $166.2 \pm 2.9 \mathrm{~b}$ & $8.58 \pm 0.10 \mathrm{ab}$ & $10.0 \pm 0.5 a$ & $224.2 \pm 2.8 b$ \\
\hline & $\mathrm{T} 4$ & $153.2 \pm 3.1 \mathrm{a}$ & $161.2 \pm 3.5 c$ & $8.74 \pm 0.10 \mathrm{ab}$ & $11.6 \pm 0.6 b$ & $194.8 \pm 2.9 c$ \\
\hline & $\mathrm{T} 5$ & $153.4 \pm 1.9 a$ & $157.6 \pm 3.7 \mathrm{c}$ & $8.58 \pm 0.16 \mathrm{ab}$ & $9.8 \pm 0.5 \mathrm{a}$ & $181.6 \pm 2.6 \mathrm{~d}$ \\
\hline & T6 & $154.8 \pm 2.5 \mathrm{a}$ & $151.8 \pm 3.1 \mathrm{c}$ & $8.50 \pm 0.10 \mathrm{ab}$ & $9.6 \pm 0.4 \mathrm{ac}$ & $178.4 \pm 2.6 \mathrm{~d}$ \\
\hline & $\mathrm{T} 7$ & $151.0 \pm 3.1 \mathrm{a}$ & $145.0 \pm 3.7 \mathrm{~d}$ & $8.42 \pm 0.11 b$ & $9.2 \pm 0.7 \mathrm{c}$ & $149.4 \pm 2.3 \mathrm{e}$ \\
\hline & T0 & $167.4 \pm 2.3 \mathrm{a}$ & $163.2 \pm 3.4 \mathrm{a}$ & $6.98 \pm 0.14 \mathrm{ab}$ & $10.0 \pm 0.4 \mathrm{ab}$ & $215.6 \pm 2.3 a$ \\
\hline & $\mathrm{T} 1$ & $165.6 \pm 3.3 \mathrm{a}$ & $165.0 \pm 2.7 \mathrm{a}$ & $7.02 \pm 0.20 \mathrm{a}$ & $10.6 \pm 0.3 \mathrm{a}$ & $215.2 \pm 2.5 a$ \\
\hline & $\mathrm{T} 2$ & $163.4 \pm 1.5 \mathrm{abc}$ & $164.4 \pm 4.2 \mathrm{a}$ & $6.82 \pm 0.10 \mathrm{abc}$ & $11.0 \pm 0.7 \mathrm{a}$ & $206.6 \pm 2.3 \mathrm{ab}$ \\
\hline \multirow{8}{*}{ Day 21} & $\mathrm{~T} 3$ & $161.4 \pm 2.9 \mathrm{abc}$ & $160.4 \pm 2.9 \mathrm{a}$ & $6.78 \pm 0.10 \mathrm{abc}$ & $11.2 \pm 0.5 \mathrm{a}$ & $200.2 \pm 2.2 b$ \\
\hline & $\mathrm{T} 4$ & $160.8 \pm 1.9 \mathrm{abc}$ & $160.2 \pm 4.5 \mathrm{ab}$ & $6.60 \pm 0.18 \mathrm{abc}$ & $11.2 \pm 0.6 \mathrm{a}$ & $199.4 \pm 2.3 b$ \\
\hline & $\mathrm{T} 5$ & $160.6 \pm 1.9 \mathrm{abc}$ & $158.0 \pm 4.7 \mathrm{~b}$ & $6.58 \pm 0.10 \mathrm{abc}$ & $9.8 \pm 0.5 b$ & $190.4 \pm 2.9 b c$ \\
\hline & T6 & $158.8 \pm 2.5 b c$ & $151.6 \pm 5.1 \mathrm{c}$ & $6.54 \pm 0.10 \mathrm{bc}$ & $9.6 \pm 0.4 b$ & $178.0 \pm 2.2 \mathrm{c}$ \\
\hline & $\mathrm{T} 7$ & $157.0 \pm 2.4 c$ & $144.4 \pm 3.7 \mathrm{~d}$ & $6.44 \pm 0.10 c$ & $8.2 \pm 0.7 c$ & $148.8 \pm 2.6 \mathrm{~d}$ \\
\hline & T0 & $156.4 \pm 2.2 \mathrm{a}$ & $159.2 \pm 3.7 \mathrm{a}$ & $6.34 \pm 0.13 a$ & $10.2 \pm 0.2 \mathrm{a}$ & $191.6 \pm 2.2 \mathrm{a}$ \\
\hline & $\mathrm{T} 1$ & $154.4 \pm 2.3 \mathrm{a}$ & $159.4 \pm 2.7 \mathrm{a}$ & $6.38 \pm 0.20 \mathrm{a}$ & $10.2 \pm 0.2 \mathrm{a}$ & $194.0 \pm 2.3 \mathrm{a}$ \\
\hline & $\mathrm{T} 2$ & $154.4 \pm 2.1 \mathrm{a}$ & $156.4 \pm 3.4 \mathrm{ab}$ & $6.18 \pm 0.10 \mathrm{ab}$ & $10.8 \pm 0.2 \mathrm{a}$ & $184.0 \pm 2.3 b$ \\
\hline \multirow{8}{*}{ Day 28} & $\mathrm{~T} 3$ & $154.2 \pm 1.5 \mathrm{a}$ & $152.8 \pm 3.1 b$ & $6.12 \pm 0.10 \mathrm{ab}$ & $11.0 \pm 0.1 b$ & $181.2 \pm 2.8 b$ \\
\hline & $\mathrm{T} 4$ & $154.4 \pm 2.9 \mathrm{a}$ & $147.4 \pm 2.9 b$ & $5.98 \pm 0.10 \mathrm{abc}$ & $11.6 \pm 0.2 b$ & $175.8 \pm 2.9 \mathrm{cb}$ \\
\hline & $\mathrm{T} 5$ & $148.6 \pm 2.5 b$ & $138.4 \pm 3.5 c$ & $5.92 \pm 0.15 \mathrm{abc}$ & $10.4 \pm 0.2 \mathrm{bac}$ & $170.6 \pm 2.8 c$ \\
\hline & T6 & $148.4 \pm 2.4 b$ & $131.6 \pm 3.7 \mathrm{~d}$ & $5.88 \pm 0.08 \mathrm{bc}$ & $10.0 \pm 0.2 \mathrm{bac}$ & $164.4 \pm 2.8 \mathrm{~d}$ \\
\hline & $\mathrm{T} 7$ & $144.0 \pm 2.4 \mathrm{~b}$ & $128.0 \pm 4.1 \mathrm{~d}$ & $5.66 \pm 0.09 c$ & $9.80 \pm 0.1 c$ & $138.4 \pm 2.2 \mathrm{e}$ \\
\hline & T0 & $150.4 \pm 2.3 \mathrm{a}$ & $153.2 \pm 3.7 \mathrm{a}$ & $5.54 \pm 0.13 a$ & $10.6 \pm 0.1 \mathrm{a}$ & $182.6 \pm 2.4 \mathrm{a}$ \\
\hline & $\mathrm{T} 1$ & $149.4 \pm 3.3 \mathrm{a}$ & $150.0 \pm 3.4 \mathrm{ab}$ & $5.58 \pm 0.20 \mathrm{a}$ & $10.2 \pm 0.2 \mathrm{a}$ & $182.2 \pm 2.2 \mathrm{a}$ \\
\hline & $\mathrm{T} 2$ & $149.6 \pm 3.1 \mathrm{a}$ & $147.8 \pm 2.7 b$ & $5.38 \pm 0.08 \mathrm{ab}$ & $10.2 \pm 0.2 \mathrm{a}$ & $171.0 \pm 2.8 \mathrm{~b}$ \\
\hline \multirow{8}{*}{ Day 35} & $\mathrm{~T} 3$ & $145.4 \pm 2.9 \mathrm{ab}$ & $142.8 \pm 2.1 b c$ & $5.32 \pm 0.10 \mathrm{ab}$ & $10.4 \pm 0.1 \mathrm{a}$ & $165.6 \pm 2.8 \mathrm{bc}$ \\
\hline & $\mathrm{T} 4$ & $145.8 \pm 2.5 \mathrm{ab}$ & $138.4 \pm 2.9 \mathrm{~cd}$ & $5.18 \pm 0.09 \mathrm{abc}$ & $11.8 \pm 0.2 b$ & $162.8 \pm 2.5 \mathrm{~cd}$ \\
\hline & T5 & $144.8 \pm 2.4 \mathrm{ab}$ & $133.0 \pm 2.5 \mathrm{de}$ & $5.12 \pm 0.15 \mathrm{abc}$ & $10.0 \pm 0.2 \mathrm{a}$ & $157.4 \pm 2.7 \mathrm{~d}$ \\
\hline & T6 & $139.8 \pm 2.2 b c$ & $130.2 \pm 2.7 \mathrm{e}$ & $5.08 \pm 0.08 b c$ & $10.0 \pm 0.1 \mathrm{a}$ & $141.4 \pm 2.7 \mathrm{e}$ \\
\hline & $\mathrm{T} 7$ & $136.0 \pm 2.3 c$ & $126.2 \pm 2.1$ & $4.84 \pm 0.08 c$ & $9.0 \pm 0.1 \mathrm{c}$ & $133.4 \pm 2.0 \mathrm{e}$ \\
\hline & T0 & $136.4 \pm 2.1 \mathrm{a}$ & $132.2 \pm 3.4 \mathrm{a}$ & $5.14 \pm 0.13 a$ & $10.8 \pm 0.1 \mathrm{a}$ & $144.6 \pm 2.5 \mathrm{a}$ \\
\hline & $\mathrm{T} 1$ & $135.4 \pm 1.5 \mathrm{a}$ & $132.0 \pm 2.7 \mathrm{a}$ & $5.16 \pm 0.20 \mathrm{a}$ & $10.6 \pm 0.2 \mathrm{a}$ & $144.2 \pm 2.4 \mathrm{a}$ \\
\hline & $\mathrm{T} 2$ & $133.4 \pm 1.9 a$ & $127.2 \pm 2.1 \mathrm{ab}$ & $4.98 \pm 0.06 \mathrm{ab}$ & $10.6 \pm 0.2 \mathrm{a}$ & $133.0 \pm 2.4 b$ \\
\hline \multirow{5}{*}{ Day 42} & T3 & $130.8 \pm 1.9 \mathrm{a}$ & $124.4 \pm 2.9 \mathrm{bc}$ & $4.92 \pm 0.10 \mathrm{ab}$ & $10.8 \pm 0.2 \mathrm{a}$ & $127.6 \pm 2.8 \mathrm{bc}$ \\
\hline & $\mathrm{T} 4$ & $129.6 \pm 2.3 a$ & $123.4 \pm 2.5 b c$ & $4.74 \pm 0.09 \mathrm{abc}$ & $12.0 \pm 0.1 b$ & $124.8 \pm 2.9 c$ \\
\hline & T5 & $121.2 \pm 2.4 b$ & $121.6 \pm 2.7 c$ & $4.72 \pm 0.15 \mathrm{abc}$ & $10.0 \pm 0.3 c$ & $119.4 \pm 2.8 \mathrm{c}$ \\
\hline & T6 & $117.8 \pm 1.5 b$ & $114.4 \pm 2.1 \mathrm{de}$ & : & $9.4 \pm 0.2 c$ & $103.4 \pm 2.9 \mathrm{~d}$ \\
\hline & $\mathrm{T} 7$ & $108.0 \pm 2.9 c$ & $110.6 \pm 3.7 \mathrm{e}$ & $4.48 \pm 0.08 c$ & $8.8 \pm 0.2 \mathrm{~d}$ & $95.4 \pm 2.2 \mathrm{~d}$ \\
\hline
\end{tabular}

Note. Chol: cholesterol, Trigl: triglyceride, ALP: alkaline phosphatase, ALT: alanine transaminase, and AST: aspartate transaminase. T0 = control 1 (without vaccination and without supplementation of $N$. sativa in diet), $\mathrm{T} 1=$ control $2(\mathrm{~T} 0+$ vaccination $), \mathrm{T} 2=\mathrm{T} 1+N$. sativa $0.5 \%, \mathrm{~T} 3=\mathrm{T} 1+N$. sativa $1 \%$, $\mathrm{T} 4=\mathrm{T} 1+N$. sativa $2 \%, \mathrm{~T} 5=\mathrm{T} 1+N$. sativa $4 \%, \mathrm{~T} 6=\mathrm{T} 1+N$. sativa $8 \%$, and $\mathrm{T} 7=\mathrm{T} 1+N$. sativa $16 \%$. Different superscript letters in each column/week indicate significant $(p<0.05)$ differences between groups, and NS stands for not significant $(p>0.05)$.

groups revealed that the decreasing effect of N. sativa on the AST level is dose-dependent and group T7 had the lowest levels during the whole experimental period. As shown in Table 6, the mean differences of the groups T6 (8\%) and T7 $(16 \%)$ significantly $(p<0.05)$ differed from those of the rest groups on day 21 of age to the end of the experimental period.

\section{Discussion}

4.1. Performance. Comparison of the vaccinated group (T1) with the unvaccinated group (T0) indicated that vaccination had a little $(p>0.05)$ adverse effect on weight gains of the chickens as expected due to vaccination stress. As shown in 
Figure 1, our results about the efficacy of $N$. sativa supplementation on the performance of broiler chickens are in agreement with the results obtained by Miraghaee et al. [24], Ghasemi et al. [38], Hossain et al. [39], and Ali et al. [47] who reported that $1 \% N$. sativa seeds in diet improved the performance of broiler chickens but there are some differences between our results with those obtained by Shewita and Taha [37] who observed a higher weight in chickens fed with $2 \%$ N. sativa seed supplementation diet when compared with the weight of those that received $10 \%$ of $N$. sativa seeds in their diet, or Durrani et al. [36] who reported a higher weight gain in $40 \mathrm{~g} / \mathrm{kg}$ (4\%) of $N$. sativa seeds in the diet. Overall, most studies indicate that a higher percentage of $N$. sativa seeds in the diet may reduce feed intake and finally affects the average weight of broiler chickens as observed in this study (Table 2$)$ for groups T6 $(8 \% \mathrm{~N}$. sativa seeds) and T7 (16\% N. sativa seeds), and the mean of the latest differed significantly $(p<0.05)$ from those of the control (T0-T1) groups (Figure 1).

4.2. Immune Response. The lack of an increase in antibody titer of chickens of the unvaccinated group (T0) during the experimental period confirmed that no environmental or cross-contamination has occurred. Lack of significant differences among treated groups during days 1-14 of age indicates that supplementation of $N$. sativa seeds on diet had no significant effects on MDA reduction of the chickens. A significant $(p=0.04)$ difference between antibody titers of group T3 and group T1 indicates that dietary inclusion of $1 \%$ $N$. sativa seeds had the highest immunomodulatory effects, while that of group T7 (16\% N. sativa seeds) had immunosuppressive effects. The results observed during this study (Figure 2) indicate that $N$. sativa is able to promote antibody response (mostly $\operatorname{IgG}$ ) in chickens as previously reported $[5,17,29,36,47,48]$. Higher antibody titers $\left(\log 2^{-7.4}\right)$ of chickens (group T3) observed during this study are in the range of expected titers that could be induced via vaccinations by two live plus one killed vaccines $[49,50]$. Our results about the efficacy of different levels of $N$. sativa seeds in the diet on immune responses of chickens also is in harmony with the results obtained by Hossain et al. [39] who reported that supplementation of $1 \%$ N. sativa seeds in a broiler diet improved the development of immunity but is in disagreement with the results obtained by Khan et al. [51]. However, our observation on antibody titers is in disagreement with those of Shewita and Taha [37] and Ghasemi et al. [38] who reported that $20 \mathrm{~g} / \mathrm{Kg}$ of $N$. sativa had the highest immunomodulatory effects, and this inconsistency could be attributed to the lack of $1 \%$ concentration in their work. Our observation on a decrease in lymphocytes percentage together with an increase in antibody titers following vaccination against Newcastle diseases is controversial to the results obtained by Islam et al. [52] who reported a decrease in antibody titer together with a rise in peripheral lymphocytes with an explanation that the numbers of antigen-specificantibody secreting B-cells were less. As recent reports indicated that $N$. sativa has induced a significant increase in antibody titers against Newcastle disease (ND) and infectious bursal disease (IBD) in broilers $[29,36]$, therefore, the reasons for this controversy results may be due to the nature of pathogenic agents, type of treatments (vaccination versus challenge), kind of experimental animal, different components and dosage of N. sativa, and the role of macrophages in the activation of Th2 cells and their roles in the activation of B-cells for further differentiation into plasma cells. For example, the increase in antibody titer against avian infectious bronchitis (IB) has been observed by Durrani et al. [36] but has not been observed by Al-Beitawi et al. [29] or various dosages of N. sativa had different effects on antibody titers against different pathogenic agents (ND, IBD, and IB) in same experimental animals "broiler chickens" [36].

4.3. Hemogram. As shown in Table 3, the lack of significant $(p>0.05)$ differences between hemogram values of the control 1 (T0) and those of control 2 (T1) groups indicates that vaccination alone did not significantly influence the hemograms. The results (Table 3 ) obtained during this study in regard to values of some hematological parameters (RBC, $\mathrm{PCV}$, and $\mathrm{Hb}$ ) in broiler chickens are in agreement with those previously reported [53].

4.4. Leukogram. Our results (Table 4) on the reduction effects of N. sativa on leukocyte counts of the chickens are in agreement with previous reports $[54,55]$. The results obtained during this study revealed that supplementation of $N$. sativa seeds in broiler's diet increased the $\mathrm{H} / \mathrm{L}$ ratio (Table 4 ), and our results are in disagreement with the results obtained by Ali et al. [26] who reported that supplementation of $1 \% N$. sativa may reduce the heterophil/lymphocyte $(\mathrm{H} / \mathrm{L})$ ratio. An increase in eosinophil values observed during this study (Table 4) is in line with the results obtained by Ahmad et al. [13] who reported that $N$. sativa rises peripheral blood lymphocytes and eosinophils in Long-Evans rats. It has been documented that monocytes-macrophages play an indispensable role in the immune system [55] and an increase in their numbers may indicate the immunomodulatory effects of $N$. sativa.

4.5. Biochemical Parameters. In general, biochemical parameters of poultry may be influenced by various factors and the results of this study on biochemical parameters (Table 5) were compared with reported reference ranges [40-42, 56-58].

4.5.1. Albumin and Total Protein. As shown in Table 5, alternation on concentrations of albumin and total protein of the chickens fed with $N$. sativa seeds observed during this study have also been reported by Miraghaee et al. [24]. However, weekly comparison (Table 5) values of total protein and albumin of the treated groups showed that differences among the groups were not significant $(p>0.05)$ as previously reported $[42,59]$ but in disagreement with the results observed by Singh and Kumar [41]. As shown in Table 5, increasing of protein and albumin concentrations of the chickens fed with $0.5-2 \%$ and decreasing of those chickens fed with $4-16 \% N$. sativa may indicate that the 
efficacy of $N$. sativa on protein and albumin concentrations is dose-dependent.

4.5.2. Glucose. The results obtained during this study (Table 5) on the level of serum glucose of chickens are in agreement with recent reports $[29,40]$ but in disagreement with other reports [41, 42]. Some controversial observations could be explained where the type of the extract, whole seed, and duration of consumption may exert different effects on glucose level as has been observed by Mohtashami [60] who reported that $N$. sativa did not significantly affect the fasting blood glucose level but significantly decreased its levels during oral glucose tolerance test (OGTT) when compared to diabetic control by affecting the time course of glucose absorption from the intestine.

4.5.3. Calcium and Phosphorus. The results obtained for calcium and phosphorus during this study (Table 5) are in agreement with previous reports that supplementation of $N$. sativa in the feed of broiler diets significantly increases the level of serum calcium $[61,62]$ because $N$. sativa possesses estrogenic activity in regulating calcium-regulating hormones [61].

4.5.4. Cholesterol and Triglyceride. Because of yolk sac in day-old chicks, the concentration of both cholesterol and triglyceride were very high at the beginning of the experiment, but as shown in Table 6, by aging, their values reduced, and their levels in the control groups at age 42 days were in the range as previously reported [63]. The results obtained during this study on the serum cholesterol and triglyceride levels of broiler chickens are in agreement with previous reports that $N$. sativa reduces serum cholesterols of chickens [21, 24, 29, 37-40, 47], in particular HDL cholesterol [26]. The reduction in serum cholesterol and triglycerides has been attributed to the lowering effect of black cumin (thymoquinone and monounsaturated fatty acids) on the synthesis of cholesterol by hepatocytes or fractional reabsorption from the small intestine [29]. It is also believed that phytosterols of $N$. sativa inhibit cholesterol absorption and may interfere with the reabsorption of endogenous cholesterol [26]. Moreover, it has also been reported that $N$. sativa decreases plasma concentrations of cholesterol by stimulating bile acid excretion [5], and a reduction in plasma cholesterol level could also be attributed to the effects of black cumin on the cholesterol excretion into the intestine $[21,26]$. As shown in Table 6, the lowering effects of N. sativa supplementation in the diet of commercial layers on cholesterol could be useful because lower egg yolk cholesterol of commercial layers' eggs is highly desirable for human consumption [5], but it may not be useful in breeders because of egg yolk role in embryo development.

4.5.5. Serum Enzymes. Serum enzymes including ALP, ALT, and AST are mainly monitored for the evaluation of liver damage [3,9], and antioxidant activity of $N$. sativa components such as thymoquinone may play a vital role in the prevention of liver tissue [64]. The reduction of ALP and AST levels of broiler chickens fed with $N$. sativa supplementation observed during this study (Table 6) is in agreement with previous reports $[21,40,59]$ but is in disagreement with that observed by Shewita and Taha [37] who reported that serum glutamic pyruvic transaminase (SGPT) level significantly increased with $N$. sativa supplementation. As shown in Table 6, increasing effects of $N$. sativa seeds on levels of ALT observed during this study have also been reported [40] and may indicate positive effects of $N$. sativa on liver health because ALT level is commonly used clinically as a biomarker for liver health.

\section{Conclusion}

Supplementation of $N$. sativa seed (1-2\%) in broiler diets, as a multipurpose natural growth promoter, improves performance, elevates humoral immune responses, affects serum biochemical profiles of broiler chickens, and induces changes in their hemogram (Hb, PCV) and leukogram, while there are no side, residual, and hazardous effects.

\section{Abbreviations}

$\begin{array}{ll}\text { N: } & \text { Nigella } \\ \text { V: } & \text { Vaccinated } \\ \text { RBC: } & \text { Red blood cells } \\ \text { PCV: } & \text { Packed cell volume } \\ \text { MCV: } & \text { Mean corpuscular volume } \\ \text { MCH: } & \text { Mean corpuscular hemoglobin } \\ \text { MCHC: } & \text { Mean corpuscular hemoglobin concentration } \\ \text { WBC: } & \text { White blood cells. }\end{array}$

\section{Data Availability}

Data used to support the findings of this study are included within the article.

\section{Conflicts of Interest}

The authors declare that there are no conflicts of interest regarding this mansucript.

\section{Acknowledgments}

This research was financially supported (grants for three DVM theses) by the Vice-Chancellor for Research of Urmia University, Urmia, Iran.

\section{References}

[1] M. Muthusamy and V. Sankar, "Phytogenic compounds used as a feed additive in poultry production," International Journal of Science, Environment and Technology, vol. 4, no. 1, pp. 167-171, 2015.

[2] M. B. Yitbarek, "Phytogenics as feed additives in poultry production: a review," International Journal of Extensive Research, vol. 3, pp. 49-60, 2015.

[3] A. A. Saleh, T. A. Ebeid, and A. M. Abudabos, "Effect of dietary phytogenics (herbal mixture) supplementation on growth performance, nutrient utilization, antioxidative 
properties, and immune response in broilers," Environmental Science and Pollution Research, vol. 25, no. 15, pp. 1460614613, 2018.

[4] S. H. Qamar, A. U. Haq, N. Asghar, S. U. Rehman, P. Akhtar, and G. Abbas, "Effect of herbal medicine supplementations (Arsilvon super, Bedgen 40 and hepa-cure herbal medicines) on growth performance, immunity and haematological profile in broilers," Advances in Zoology and Botany, vol. 3, no. 2, pp. 17-23, 2015.

[5] T. Azeem, Z. Ur-Rehman, S. Umar, M. Asif, M. Arif, and A. Rahman, "Effect of Nigella Sativa on poultry health and production: a review," Science Letters, vol. 2, no. 2, pp. 76-82, 2014.

[6] S. Nazir, S. Arif Zaidi, and Z. Zaidi, "Kalonji seeds (Nigella sativa) in strengthening the immune system," Madridge Journal of Case Reports and Studies, vol. 2, no. 1, pp. 55-56, 2018.

[7] M. T. Salman, R. A. Khan, and I. Shukla, "Antibacterial Activity of Nigella Sativa Linn. seeds against multiple antibiotics resistant clinical strains of Staphylococcus aureus," International Archives of BioMedical and Clinical Research, vol. 2, no. 3, pp. 96-99, 2016.

[8] G. Z. A. Soliman, A. M. Hashem, and M. Arafa, "Protective effect of Curcuma longa or Nigella sativa on AflatoxinB $B_{1}$ induced hepato-toxicity in rats in relation to food safety on public health," Medical Journal of Cairo University, vol. 80, no. 2, pp. 191-203, 2012.

[9] H. Pourbakhsh, E. Taghiabadi, K. Abnous, A. T. Hariri, S. M. Hosseini, and H. Hosseinzadeh, "Effect of Nigella sativa fixed oil on ethanol toxicity in rats," Iranian Journal of Basic Medical Sciences, vol. 17, no. 12, pp. 1020-1031, 2014.

[10] R. D. M. El-Shoukary, M. H. A. Darwish, and M. A. M. AbdelRahman, "Behavioral, performance, carcass traits and hormonal changes of heat stressed broilers feeding black and coriander seeds," Journal of Advanced Veterinary Research, vol. 4, no. 1, pp. 93-101, 2014.

[11] M. R. Haj-Syed Hadi, M. T. Darzi, and G.-H. Riazi, "Black cumin (Nigella sativa L.) yield affected by irrigation and plant growth promoting bacteria," Journal of Medicinal Plants and By-Products, vol. 2, pp. 125-133, 2016.

[12] Y. Niu, L. Zhou, L. Meng et al., "Recent progress on chemical constituents and pharmacological effects of the genus Nigella," Evidence-Based Complementary and Alternative Medicine, vol. 2020, Article ID 6756835, 15 pages, 2020.

[13] A. Ahmad, A. Husain, M. Mujeeb et al., "A review on therapeutic potential of Nigella sativa: a miracle herb," Asian Pacific Journal of Tropical Biomedicine, vol. 3, no. 5, pp. 337-352, 2013.

[14] H. Mollazadeh and H. Hosseinzadeh, "The protective effect of Nigella sativa against liver injury: a review," Iranian Journal of Basic Medical Sciences, vol. 17, pp. 958-966, 2014.

[15] I. Jehani and E. H. Abdel-Hakiem, "Quality improvement of damietta cheese using some spices extract," Assiut Veterinary Medical Journal, vol. 61, no. 147, pp. 24-32, 2015.

[16] M. Ardiana, B. S. Pikir, A. Santoso, H. O. Hermawan, and M. J. Al-Farabi, "Effect of Nigella sativa supplementation on oxidative stress and antioxidant parameters: a Meta-Analysis of Randomized Controlled Trials," TheScientificWorldJournalfic World Journal, vol. 2020, Article ID 2390706, 7 pages, 2020.

[17] F. Ahmad, F. A. Ahmad, S. A. Ashraf et al., "An updated knowledge of Black seed (Nigella sativa Linn): review of phytochemical constituents and pharmacological properties," Journal of Herbal Medicine, vol. 25, Article ID 100404, 2021.
[18] M. N. Islam, K. S. Hossain, P. P. Sarker et al., "Revisiting pharmacological potentials of Nigella sativa seed: a promising option for COVID-19 prevention and cure," Phytotherapy Research, vol. 35, no. 3, pp. 1329-1344, 2020.

[19] M. F.-E.-A. Kulyar, R. Li, K. Mehmood, M. Waqas, K. Li, and J. Li, "Potential influence of Nigella sativa (Black cumin) in reinforcing immune system: a hope to decelerate the COVID19 pandemic," Phytomedicine, vol. 85, Article ID 153277, 2021.

[20] N. M. P. Maideen, "Prophetic medicine-Nigella Sativa (Black Cumin seeds) potential herb for COVID-19?" Journal of Pharmacopuncture, vol. 23, no. 2, pp. 62-70, 2020.

[21] A. Saleh, "Nigella seed oil as alternative to avilamycin antibiotic in broiler chicken diets," South African Journal of Animal Science, vol. 44, no. 3, pp. 254-261, 2014.

[22] R. Aydin, M. Karaman, T. Cicek, and H. yardibi, "Black cumin (Nigella sativa L.) supplementation into the diet of the laying hen positively influences egg yield parameters, shell quality, and decreases egg cholesterol," Poultry Science, vol. 87, no. 12, pp. 2590-2595, 2008.

[23] J. Boka, A. H. Mahdavi, A. H. Samie, and R. Jahanian, "Effect of different levels of black cumin (Nigella sativa L.) on performance, intestinal Escherichia coli colonization and jejunal morphology in laying hens," Journal of Animal Physiology and Animal Nutrition, vol. 98, no. 2, pp. 373-383, 2014.

[24] S. S. Miraghaee, B. Heidary, H. Almasi, A. Shabani, M. Elahi, and M. H. Modaber-Nia, "The effects of Nigella sativa powder (black seed) and Echinacea purpurea (L.) Moench extract on performance, some blood biochemical and hematological parameters in broiler chickens," African Journal of Biotechnology, vol. 10, no. 82, pp. 19249-19254, 2011.

[25] A. Ragab, K. El-Reidy, and H. Gaafar, "Effect of diet supplemented with pumpkin (cucurbita moschata) and black seed (Nigella sativa) oils on performance of rabbits: 1- growth performance, blood hematology and carcass traits of growing rabbits," Journal of Animal and Poultry Production, vol. 4, no. 7, pp. 381-393, 2013.

[26] O. A. A. Ali, N. Suthama, and L. D. Mahfud, "The effect of feeding Black Cumin (Nigella sativa) and Vitamin C on blood lipid profiles and growth performance of broilers," International Refereed Journal of Engineering and Science, vol. 3, no. 4, pp. 28-33, 2014.

[27] G. Elmowalid, A. M. Amar, and A. A. M. Ahmad, "Nigella sativa seed extract: 1. Enhancement of sheep macrophage immune functions in vitro," Research in Veterinary Science, vol. 95, no. 2, pp. 437-443, 2013.

[28] S. Büyüköztürk, A. Gelincik, F. Özşeker et al., "Nigella sativa (black seed) oil does not affect the T-helper 1 and T-helper 2 type cytokine production from splenic mononuclear cells in allergen sensitized mice," Journal of Ethnopharmacology, vol. 100, no. 3, pp. 295-298, 2005.

[29] N. A. Al-Beitawi, S. S. El-Ghousein, and A. H. Nofal, "Replacing bacitracin methylene disalicylate by crushed Nigella sativa seeds in broiler rations and its effects on growth, blood constituents and immunity," Livestock Science, vol. 125, no. 2-3, pp. 304-307, 2009.

[30] K. Dhama, S. K. Latheef, S. Mani et al., "Multiple beneficial applications and modes of action of herbs in poultry health and production-A review," International Journal of Pharmacology, vol. 11, no. 3, pp. 152-176, 2015.

[31] A. R. Gado, H. F. Ellakany, A. R. Elbestawy et al., "Herbal medicine additives as powerful agents to control and prevent avian influenza virus in poultry-a review," Annals of Animal Science, vol. 19, no. 4, pp. 905-935, 2019. 
[32] I. M. Yimer, K. B. Tuem, A. karim, N. Ur-Rehman, and F. Anwar, "Nigella sativa L. (black cumin): a promising natural remedy for wide range of illnesses," Evidence-Based Complementary and Alternative, vol. 2019, Article ID 1528635, 17 pages, 2019.

[33] M. N. Alloui, A. Agabou, and N. Alloui, "Application of herbs and phytogenic feed additives in poultry production-A Review," Global Journal of Animal Scientific Research, vol. 2, no. 3, pp. 234-243, 2014.

[34] M. Saeed, A. Baloch, M. Wang et al., "Use of Cichorium Intybus leaf extract as growth promoter, hepatoprotectant and immune modulent in broilers," Journal of Animal Production Advances, vol. 5, no. 1, pp. 585-591, 2015.

[35] C. Yang, M. A. Chowdhury, Y. Huo, and J. Gong, "Phytogenic compounds as alternatives to in-feed antibiotics: potentials and challenges in application," Pathogens, vol. 4, no. 1, pp. 137-156, 2015.

[36] F. R. Durrani, "Effect of different levels of feed added black seed (Nigella sativa L.) on the performance of broiler chicks," Pakistan Journal of Biological Sciences, vol. 10, no. 22, pp. 4164-4167, 2007.

[37] R. S. Shewita and A. F. Taha, "Effect of dietary supplementation of different levels of black seed (Nigella sativa L.) on growth performance, hematological and carcass parameters of broiler chicks," International Scholarly and Scientific Research \& Innovation, vol. 5, no. 5, pp. 1031-1037, 2011.

[38] H. A. Ghasemi, N. Kasani, and K. Taherpour, "Effects of black cumin seed (Nigella sativa L.), a probiotic, a prebiotic and a synbiotic on growth performance, immune response and blood characteristics of male broilers," Livestock Science, vol. 164, pp. 128-134, 2014.

[39] M. M. Hossain, A. J. Howlader, M. N. Islam, and M. A. Beg, "Evaluation of locally available herbs and spices on physical, biochemical and economical parameters on broiler production," International Journal of Plant, Animal and Environmental Sciences, vol. 4, no. 1, pp. 317-322, 2014.

[40] K. Shirzadegan, P. Fallahpour, I. Nickkhah, and H. I. Taheri, "Black Cumin (Nigella sativa) supplementation in the diet of broilers influences liver weight and its enzymes," Iranian Journal of Applied Animal Science, vol. 5, no. 1, pp. 173-178, 2015.

[41] P. K. Singh and A. Kumar, "Effect of dietary Black Cumin (Nigella sativa) on the growth performance, nutrient utilization, blood biochemical profile and carcass traits in broiler chickens," Animal Nutrition and Feed Technology, vol. 18, no. 3, pp. 409-419, 2018.

[42] S. Aydogan, "The effect of dietary garlic (allium sativum), black cumin (Nigella sativa) and their combination on performance, intestine morphometry, serum biochemistry and antioxidant status of broiler chickens," Brazilian Journal of Poultry Science, vol. 22, no. 4, 2020.

[43] P. J. Miller, C. L. Afonso, J. El Attrache et al., "Effects of Newcastle disease virus vaccine antibodies on the shedding and transmission of challenge viruses," Developmental \& Comparative Immunology, vol. 41, no. 4, pp. 505-513, 2013.

[44] P. J. Miller and G. Koch, "Newcastle disease," in Diseases of Poultry, S. E. Swayne, Ed., , John Wiley \& Son, In, Ames, IW, USA, 2013, https://onlinelibrary.wiley.com/doi/book/10. 1002/9781119371199, 14th edition.

[45] S. E. Collett and J. Smith, "Principles of disease prevention, diagnosis, and control," in Diseases of Poultry, S. E. Swayne, Ed., Wiley-Blackwell, Oxford, UK, 14th edition, 2020.

[46] T. W. Campbell, Avian Hematology and Cytology, Iowa State University Press, Ames, IW, USA, 1992.
[47] S. Ali, M. Mukhtar, S. Manzoor et al., "Effect of garlic, black seed and turmeric on the growth of broiler chicken," Pakistan Journal of Nutrition, vol. 13, no. 4, pp. 204-210, 2014.

[48] F. K. Abdullah, A. Y. Al-Nasser, A. Al-Saffar, A. E. Omar, and G. Ragheb, "Impact of dietary supplementation of different levels of black seeds (Nigella sativa L.) on production performance, mortality and immunity of broiler chickens," International Journal of Poultry Science, vol. 18, no. 10, pp. 467-474, 2019.

[49] Office International Epizootic (OIE), "Newcastle disease," in Terrestrial Manual 2012OIE, Paris, France, 2012, https://www. oie.int/fileadmin/home/eng/animal_health_in_the_world/ docs/pdf/.

[50] S. R. Rehman, K. Muhammad, T. Yaqub, M. S. Khan, K. Hanif, and R. Yasmeen, "Antimicrobial activity of mentofin and its effect on antibody response of broilers to Newcastle disease virus vaccine," The Journal of Animal \& Plant Sciences, vol. 23, no. 4, pp. 1008-1011, 2013.

[51] S. H. Khan, M. A. Anjum, A. Parveen, T. Khawaja, and N. M. Ashraf, "Effects of black cumin seed (Nigella sativa L.) on performance and immune system in newly evolved crossbred laying hens," Veterinary Quarterly, vol. 33, no. 1, pp. 13-19, 2013.

[52] S. Nazrul Islam, P. Begum, T. Ahsan, S. Huque, and M. Ahsan, "Immunosuppressive and cytotoxic properties ofNigella sativa," Phytotherapy Research, vol. 18, no. 5, pp. 395-398, 2004.

[53] N. A. Al-Beitawi, S. S. El-Ghousein, S. Safaa, and M. Z. Athamneh, "Effect of adding crushed Pimpinella anisum, Nigella sativa seeds and Thymus vulgaris mixture to antibiotics-free rations of vaccinated and non-vaccinated male broilers on growth performance, antibody titer and haematological profile," Italian Journal of Animal Science, vol. 9, no. 2, p. e43, 2010.

[54] M. L. Salem, "Immunomodulatory and therapeutic properties of the Nigella sativa L. seed," International Immunopharmacology, vol. 5, no. 13-14, pp. 1749-1770, 2005.

[55] W.-T. Ma, F. Gao, K. Gu, and D.-K. Chen, "The role of monocytes and macrophages in autoimmune diseases: a comprehensive review," Frontiers in Immunology, vol. 10, p. 1140, 2019.

[56] S. Simaraks, O. Chinrasri, and W. Aengwanich, "Hematological, electrolyte and serum biochemical values of the Thai indigenous chickens (Gallus domesticus) in northeastern, Thailand," Songklanakarin Journal of Science and Technology, vol. 26, no. 3, pp. 425-430, 2004.

[57] Z. Nasir, "Comparison of effects of Echinacea purpurea juices and Nigella sativa seeds on performance, some blood parameters, carcass and meat quality of broilers," Doctoral dissertation, University of Hohenheim, Stuttgart, Germany, 2009.

[58] O. Alabi, F. Aderemi, and O. Adeniji, "Effect of alternative housing Systems on blood profile of egg-type chickens in humid tropics," American Journal of Experimental Agriculture, vol. 7, no. 4, pp. 197-204, 2015.

[59] K. Al-Kubaisy and M. Al-Noaemi, "A protective role of Nigella sativa oil against the harmful effect of $\mathrm{CCl} 4$ on the liver cells," The Internet Journal of Nutrition and Wellness, vol. 3, no. $1,2006$.

[60] A. Mohtashami, "Effects of bread with Nigella sativa on blood glucose, blood pressure and anthropometric indices in patients with metabolic syndrome," Clinical Nutrition Research, vol. 8, no. 2, pp. 138-147, 2019.

[61] M. A. Dollah, S. Parhizkar, L. A. Lattif, A. S. ardekani, and D. E. Zulaikha, "Natural approach for Calcium regulating 
hormone maintenance: beneficial effects of Black seed," Asian Journal of Biomedical and Pharmaceutical Sciences, vol. 2, no. 15, pp. 32-37, 2012.

[62] P. R. Saunders, "Nigella sativa dissolves small renal liths," Natural Medicine Journal, vol. 11, no. 12, 2019.

[63] U. Polat, D. Yesilbag, and M. Eren, "Serum biochemical profile of broiler chickens fed diets containing rosemary and rosemary volatile oil," Journal of Biological \& Environmental Sciences, vol. 5, no. 13, pp. 23-30, 2011.

[64] F. Yildiz, S. Coban, A. Terzi et al., "Nigella sativa relieves the deleterious effects of ischemia reperfusion injury on liver," World Journal of Gastroenterology, vol. 14, no. 33, pp. 52045209, 2008. 